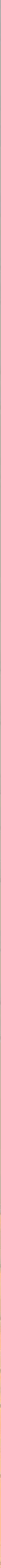




\section{Irreducibility of the Gorenstein loci of Hilbert schemes via ray families}

\section{Gianfranco Casnati, Joachim Jelisiejew and Roberto Notari}

We analyze the Gorenstein locus of the Hilbert scheme of $d$ points on $\mathbb{P}^{n}$ i.e., the open subscheme parameterizing zero-dimensional Gorenstein subschemes of $\mathbb{P}^{n}$ of degree $d$. We give new sufficient criteria for smoothability and smoothness of points of the Gorenstein locus. In particular we prove that this locus is irreducible when $d \leq 13$ and find its components when $d=14$.

The proof is relatively self-contained and it does not rely on a computer algebra system. As a by-product, we give equations of the fourth secant variety to the $d$-th Veronese reembedding of $\mathbb{P}^{n}$ for $d \geq 4$.

1. Introduction

2. Preliminaries

3. Standard form of the dual generator

4. Special forms of dual socle generators

5. Ray sums, ray families and their flatness

6. Proof of the main theorem and comments on the degree 14 cas $\$ 557$

List of symbols

1567

Acknowledgements

1568

References

A list of symbols can be found on page 1567.

Casnati and Notari are supported by the framework of PRIN 2010/11 "Geometria delle varietà algebriche", cofinanced by MIUR, and are members of GNSAGA of INdAM. Jelisiejew was partially supported by the project "Secant varieties, computational complexity, and toric degenerations" realized within the Homing Plus programme of Foundation for Polish Science, cofinanced by the European Union, Regional Development Fund. Jelisiejew is a doctoral fellow at the Warsaw Center of Mathematics and Computer Science financed by the Polish program KNOW. This paper is a part of the "Computational complexity, generalized Waring type problems and tensor decompositions" project within "Canaletto", the executive program for scientific and technological cooperation between Italy and Poland, 2013-2015.

MSC2010: primary 14C05; secondary 13H10, 14D15.

Keywords: Hilbert scheme of points, smoothability, Gorenstein algebra, secant variety. 


\section{Introduction}

Let $k$ be an algebraically closed field of characteristic neither 2 nor 3 and denote by $\mathcal{H}_{\text {ilb }}{ }_{p(t)} \mathbb{P}^{N}$ the Hilbert scheme parameterizing closed subschemes in $\mathbb{P}^{N}$ with fixed Hilbert polynomial $p(t) \in \mathbb{Q}[t]$. Since A. Grothendieck proved the existence of such a parameter space in 1966 (see [Grothendieck 1995]), the problem of dealing with $\mathcal{H i l b}_{p(t)} \mathbb{P}^{N}$ and its subloci has been a fruitful field, attracting the interest of many researchers in algebraic geometry.

Only to quickly mention some of the classical results which deserve, in our opinion, particular attention, we recall Hartshorne's [1966] proof of the connectedness of $\mathcal{H i l b}_{p(t)} \mathbb{P}^{N}$, the description of the locus of codimension-2, arithmetically Cohen-Macaulay subschemes due to J. Fogarty [1968] (for the dimension-zero case) and G. Ellingsrud [1975] (for higher dimensions) and of the study of the locus of codimension-3, arithmetically Gorenstein subschemes due to J. Kleppe and R. M. Miró-Roig [Miró-Roig 1992; Kleppe and Miró-Roig 1998].

If we restrict our attention to the case of zero-dimensional subschemes of degree $d$, i.e., subschemes with Hilbert polynomial $p(t)=d$, then the first significant results are due to Fogarty [1968] and to A. Iarrobino [1972].

Fogarty [1968] proves that $\mathcal{H i l b}_{d} \mathbb{P}^{2}$ is smooth, hence irreducible thanks to Hartshorne's connectedness result (the same result holds when one substitutes $\mathbb{P}^{2}$ by any smooth surface).

On the other hand, Iarrobino [1972] deals with the reducibility when $N \geq 3$ and $d$ is large with respect to $N$. In order to better understand the result, recall that the locus of reduced schemes $\mathcal{R} \subseteq \mathcal{H i l b}_{d} \mathbb{P}^{N}$ is birational to a suitable open subset of the $d$-th symmetric product of $\mathbb{P}^{N}$, thus it is irreducible of dimension $d N$. We will denote by $\mathcal{H i l b}_{d}^{\text {gen }} \mathbb{P}^{N}$ its closure in $\mathcal{H i l b}_{d} \mathbb{P}^{N}$. It is a well-known and easy fact that $\mathcal{H i l b}_{d}^{\text {gen }} \mathbb{P}^{N}$ is an irreducible component of dimension $d N$, by construction. Iarrobino [1972] proves that $\mathcal{H i l b}_{d} \mathbb{P}^{N}$ is never irreducible when $d \gg N \geq 3$, showing that there is a family of schemes of dimension greater than $d N$. Such a family is thus necessarily contained in a component different from $\mathcal{H i l b}_{d}^{\text {gen }} \mathbb{P}^{N}$.

D. A. Cartwright, D. Erman, M. Velasco and B. Viray [Cartwright et al. 2009] proved that already for $d=8$ and $N \geq 4$, the scheme $\mathcal{H i l b}_{d} \mathbb{P}^{N}$ is reducible.

In view of these earlier works it seems reasonable to consider the irreducibility and smoothness of open loci in $\mathcal{H i l b}_{d} \mathbb{P}^{N}$ defined by particular algebraic and geometric properties. In the present paper we are interested in the locus $\mathcal{H} i l b_{d}^{G} \mathbb{P}^{N}$ of points in $\mathcal{H}_{i l b} \mathbb{P}^{N}$ representing schemes which are Gorenstein. This is an important locus, e.g., it has an irreducible component $\mathcal{H i l b}_{d}^{G, \text { gen }} \mathbb{P}^{N}:=\mathcal{H i l b}_{d}^{\text {gen }} \mathbb{P}^{N} \cap \mathcal{H} i l b_{d}^{G} \mathbb{P}^{N}$ of dimension $d N$ containing all the points representing reduced schemes. Moreover, it is open, but in general not dense, in $\mathcal{H i l b}_{d} \mathbb{P}^{N}$. Recently, interesting interactions between $\mathcal{H i l b}_{d}^{G} \mathbb{P}^{N}$ and the geometry of secant varieties and general topology have 
been found (see, for example, [Buczyńska and Buczyński 2014; Buczyński et al. $\geq 2015]$ ).

Some results about $\mathcal{H i l b}{ }_{d}^{G} \mathbb{P}^{N}$ are known. The irreducibility and smoothness of $\mathcal{H}$ ilb ${ }_{d}^{G} \mathbb{P}^{N}$ when $N \leq 3$ is part of folklore (see [Casnati and Notari 2009, Corollary 2.6] for more precise references). When $N \geq 4$, the properties of $\mathcal{H i l b}_{d}^{G} \mathbb{P}^{N}$ have been the subject of intensive study in recent years.

For example, it is classically known that $\mathcal{H i l b}_{d}^{G} \mathbb{P}^{N}$ is never irreducible for $d \geq 14$ and $N \geq 6$, at least when the characteristic of $k$ is zero (see [Iarrobino and Emsalem 1978; Iarrobino and Kanev 1999]; see also [Casnati and Notari 2011]). Also, for $N=4$ and $d \geq 140$, or $N=5$ and $d \geq 42$, the scheme $\mathcal{H i l b}_{d}^{G} \mathbb{P}^{N}$ is reducible; see [Buczyńska and Buczyński 2014, Section 6, p. 81]. For fixed $N \in\{4,5\}$, the minimal value of $d$ for which this scheme is reducible is not known.

As reflected by the above references, it is natural to ask whether $\mathcal{H i l b}_{d}^{G} \mathrm{P}^{N}$ is irreducible when $d \leq 13$ and $N$ is arbitrary. There is some evidence of an affirmative answer to this question. Indeed, Casnati and Notari [2009; 2011; 2014a; 2014b] studied the locus $\mathcal{H i l b}_{d}^{G} \mathbb{P}^{N}$ when $d \leq 11$ and $N$ is arbitrary, proving its irreducibility and dealing in detail with its singular locus in a series of papers.

A key point in the study of a zero-dimensional scheme $X \subseteq \mathbb{P}^{N}$ is that it is abstractly isomorphic to $\operatorname{Spec} A$, where $A$ is an Artin $k$-algebra with $\operatorname{dim}_{k}(A)=d$. Moreover, the irreducible components of such an $X$ correspond bijectively to those direct summands of $A$, which are local. Thus, in order to deal with $\mathcal{H i l b}_{d} \mathbb{P}^{N}$, it suffices to deal with the irreducible schemes in $\mathcal{H i l b}_{d^{\prime}} \mathbb{P}^{N}$ for each $d^{\prime} \leq d$.

In all of the aforementioned papers, the methods used in the study of $\mathcal{H i l b}_{d}^{G} \mathbb{P}^{N}$ rely on an almost explicit classification of the possible structure of local, Artin, Gorenstein $k$-algebras of length $d$. Once such a classification is obtained, the authors prove that all the corresponding irreducible schemes are smoothable, i.e., actually

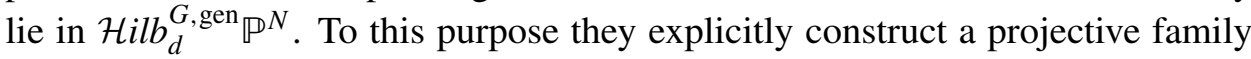
flatly deforming the scheme they are interested in (or, equivalently, the underlying algebra) to reducible schemes that they know to be in $\mathcal{H i l b}_{d}^{G, \text { gen }} \mathbb{P}^{N}$ because their components have lower degree.

Though such an approach sometimes seems to be too heavy in terms of calculations, it is only thanks to such a partial classification that it is possible to state precise results about the singularities of $\mathcal{H i l b}_{d}^{G} \mathbb{P}^{N}$.

However, there are families $H_{d}$ of schemes of degree $d=10$ and 11 for which an explicit algebraic description in the above sense cannot be obtained (see Section 3 of [Casnati and Notari 2011] for the case $d=10$ and Section 4 of [Casnati and Notari 2014b] for $d=11$ ). Nevertheless, using an alternative approach, the authors are still able to prove the irreducibility of $\mathcal{H i l b}_{d}^{G} \mathbb{P}^{N}$ and study its singular locus. Indeed, using Macaulay's theory of inverse systems, the authors check the irreducibility of 
the aforementioned loci $H_{d}$ inside $\mathcal{H} i b_{d}^{G} \mathbb{p}^{N}$. Then they show the existence of a smooth point in $H_{d} \cap \mathcal{H i l b}{ }_{d}^{G, \text { gen }} \mathbb{P}^{N}$. Hence, it follows that $H_{d} \subseteq \mathcal{H} i l b_{d}^{G, g e n} \mathbb{P}^{N}$.

The aim of the present paper is to refine and generalize this method. First, we avoid a case-by-case approach by analyzing large classes of algebras. Second, in [Casnati and Notari 2011; 2014b] a direct check (e.g., using a computer algebra program) is required to compute the dimension of the tangent space to the Hilbert scheme at some specific points to conclude that they are smooth. We avoid the need for such computations by exhibiting classes of points which are smooth, making the paper self-contained.

Using this method, we finally prove the following two statements:

Theorem A. If the characteristic of $k$ is neither 2 nor 3 , then $\mathcal{H i l b}_{d}^{G} \mathbb{P}^{N}$ is irreducible of dimension $d N$ for each $d \leq 13$ and for $d=14$ and $N \leq 5$.

Theorem B. If the characteristic of $k$ is 0 and $N \geq 6$, then $\mathcal{H i l b}_{14}^{G} \mathbb{P}^{N}$ is connected and it has exactly two irreducible components, which are generically smooth.

Theorem A has an interesting consequence regarding secant varieties of Veronese embeddings. Geramita [1999] conjectures that the ideal of the 2-nd secant variety (the variety of secant lines) of the $d$-th Veronese embedding of $\mathbb{P}^{n}$ is generated by the $3 \times 3$ minors of the $i$-th catalecticant matrix for $2 \leq i \leq d-2$. This conjecture was confirmed in [Raicu 2012]. As pointed out in [Buczyńska and Buczyński 2014, Section 8.1], Theorem A allows us to extend the above result as follows: if $r \leq 13$ and $2 r \leq d$, then for every $r \leq i \leq d-r$ the set-theoretic equations of the $r$-th secant variety of the $d$-th Veronese embedding of $\mathbb{P}^{n}$ are given by the $(r+1) \times(r+1)$ minors of the $i$-th catalecticant matrix.

The proofs of Theorem A and Theorem B are highly interlaced and they follow from a long series of partial results. In order to better explain the ideas and methods behind their proofs, we will describe in the following lines the structure of the paper.

In our analysis we incorporate several tools. In Section 2 we recall the classical ones, most notably Macaulay's correspondence for local, Artinian, Gorenstein algebras and Macaulay's growth theorem. Moreover, we also list some criteria for checking the flatness of a family of algebras which will be repeatedly used throughout the whole paper.

In Section 3 we analyze Artin Gorenstein quotients of a power series ring and exploit the rich automorphism group of this ring to put the quotient into suitable standard form, extending a result of Iarrobino.

In Section 4 we further analyze the quotients, especially their dual socle generators. We also construct several irreducible subloci of the Hilbert scheme using the theory of secant varieties. We make a small contribution to this theory, showing 
that the fourth secant variety to a Veronese reembedding of $\mathbb{P}^{n}$ is defined by minors of a suitable catalecticant matrix.

Section 5 introduces a central object in our study: a class of families, called ray families, for which we have relatively good control of the flatness and, in special cases, fibers. Most notably, Section 5B gives a class of tangent-preserving flat families, which enable us to construct smooth points on the Hilbert scheme of points without the necessity of heavy computations.

Finally, in Section 6, we give the proofs of Theorems A and B. It is worth mentioning that these results are rather easy consequences of the introduced machinery. We also prove the following general smoothability result (see Theorem 6.14), which has no restriction on the length of the algebra and generalizes the smoothability results from [Sally 1979; Casnati and Notari 2014a; Elias and Valla 2011].

Theorem C. Let $k$ be an algebraically closed field of characteristic neither 2 nor 3 . Let $A$ be a local Artin Gorenstein k-algebra with maximal ideal $\mathfrak{m}$.

If $\operatorname{dim}_{k}\left(\mathfrak{m}^{2} / \mathfrak{m}^{3}\right) \leq 5$ and $\operatorname{dim}_{k}\left(\mathfrak{m}^{3} / \mathfrak{m}^{4}\right) \leq 2$, then $\operatorname{Spec} A$ is smoothable.

\section{Preliminaries}

Let $n$ be a natural number. We let $\left(S, \mathfrak{m}_{S}, k\right)$ be the power series ring $k \llbracket \alpha_{1}, \ldots, \alpha_{n} \rrbracket$ of dimension $n$ with a fixed basis $\alpha_{1}, \ldots, \alpha_{n}$. The basis chosen determines a polynomial ring $S_{\text {poly }}=k\left[\alpha_{1}, \ldots, \alpha_{n}\right] \subseteq S$. By $P$ we denote the polynomial ring $k\left[x_{1}, \ldots, x_{n}\right]$. We will later define a duality between $S$ and $P$; see Section 2B. We usually think of $n$ being large enough, so that the considered local Artin algebras are quotients of $S$.

For $f \in P$, we say that $f$ does not contain $x_{i}$ if $f \in k\left[x_{1}, \ldots, x_{i-1}, x_{i+1}, \ldots, x_{n}\right]$; and similarly for $\sigma \in S$ or $\sigma \in S_{\text {poly }}$. For $f \in P$, by $f_{d}$ we denote the degree- $d$ part of $f$, with respect to the total degree; and similarly for $\sigma \in S$.

By $P_{m}$ and $P_{\leq m}$ we denote the space of homogeneous polynomials of degree $m$ and (not necessarily homogeneous) polynomials of degree at most $m$, respectively. These spaces are naturally affine spaces over $k$, which equips them with a scheme structure.

Recall that $S$ has a rich automorphism group: for every choice of elements $\sigma_{1}, \ldots, \sigma_{n} \in \mathfrak{m}_{S}$ that are linearly independent in $\mathfrak{m}_{S} / \mathfrak{m}_{S}^{2}$, there is a unique automorphism $\varphi$ of $S$ such that $\varphi\left(\alpha_{i}\right)=\sigma_{i}$ for $i=1,2, \ldots, n$. The existence of such automorphisms is employed in Section 4 to put the considered Artin Gorenstein algebras in a better form. See, e.g., [Elias and Rossi 2015, Section 2] for details and examples of this method.

Remark 2.1. For the reader's convenience, we introduce numerous examples, which illustrate the possible applications. In all these examples $k$ may have arbitrary characteristic except 2 or 3 , unless otherwise stated. 
2A. Artin Gorenstein schemes and algebras. In this section we recall the basic facts about Artin Gorenstein algebras. For a more thorough treatment we refer to [Iarrobino and Kanev 1999; Eisenbud 1995; Casnati and Notari 2009; Jelisiejew 2014a].

Finite-type, zero-dimensional schemes correspond to Artin algebras. Every such algebra $A$ splits as a finite product of its localizations at maximal ideals, which corresponds to the fact that the support of $\operatorname{Spec} A$ is finite and totally disconnected. Therefore, we will focus our interest on local Artin $k$-algebras. Since $k$ is algebraically closed, such algebras have residue field $k$.

An important invariant of a local algebra $(A, \mathfrak{m}, k)$ is its Hilbert function $H_{A}$, defined by $H_{A}(l)=\operatorname{dim}_{k} \mathfrak{m}^{l} / \mathfrak{m}^{l+1}$. Since $H_{A}(l)=0$ for $l \gg 0$, it is usual to write $H_{A}$ as the vector of its nonzero values. The socle degree of $A$ is the largest $l$ such that $H_{A}(l) \neq 0$. Such an algebra is Gorenstein if the annihilator of $\mathfrak{m}$ is a one-dimensional vector space over $k$; see [Eisenbud 1995, Chapter 21].

We recall for the reader's benefit that a finite, not necessarily local, algebra $A$ is Gorenstein if and only if all its localizations at maximal ideals are Gorenstein (in particular, it is meaningful to discuss the irreducibility of the Gorenstein locus in the Hilbert scheme by reducing to the study of deformations of local Gorenstein algebras; see Section 2D).

Since $k$ is algebraically closed, we may write each Artin local algebra $(A, \mathfrak{m}, k)$ as a quotient of the power series ring $S=k \llbracket \alpha_{1}, \ldots, \alpha_{n} \rrbracket$ when $n$ is large enough; in fact, $n \geq H_{A}(1)$ is sufficient. Since $\operatorname{dim}_{k} A$ is finite, such a presentation gives a presentation $A=S_{\text {poly }} / I$, i.e., a point $[\operatorname{Spec} A]$ of the Hilbert scheme of $\mathbb{A}^{n}=\operatorname{Spec} S_{\text {poly }}$.

2B. Contraction map and apolar algebras. In this section we introduce the contraction mapping, which is closely related to Macaulay's inverse systems. We refer to [Iarrobino 1994; Eisenbud 1995, Chapter 21] for details and proofs.

Recall that $P=k\left[x_{1}, \ldots, x_{n}\right]$ is a polynomial ring and $S=k \llbracket \alpha_{1}, \ldots, \alpha_{n} \rrbracket$ is a power series ring. The $k$-algebra $S$ acts on $P$ by contraction (see [Iarrobino and Kanev 1999, Definition 1.1]). This action is denoted by $(\cdot)\lrcorner(\cdot): S \times P \rightarrow P$ and defined as follows. Let $\boldsymbol{x}^{\boldsymbol{a}}=x_{1}^{a_{1}} \cdots x_{n}^{a_{n}} \in P$ and $\boldsymbol{\alpha}^{\boldsymbol{b}}=\alpha_{1}^{b_{1}} \cdots \alpha_{n}^{b_{n}} \in S$ be monomials. We write $\boldsymbol{a} \geq \boldsymbol{b}$ if and only if $a_{i} \geq b_{i}$ for all $1 \leq i \leq n$. Then

$$
\left.\alpha^{b}\right\lrcorner x^{a}:= \begin{cases}x^{a-b} & \text { if } a \geq b, \\ 0 & \text { otherwise. }\end{cases}
$$

This action extends to $S \times P \rightarrow P$ by $k$-linearity on $P$ and countable $k$-linearity on $S$.

The contraction action induces a perfect pairing between $S / \mathfrak{m}_{S}^{s+1}$ and $P_{\leq s}$, which restricts to a perfect pairing between the degree-s polynomials in $S_{\text {poly }}$ and $P$. These pairings are compatible for different choices of $s$. 
If $f \in P$ then a derivative of $f$ is an element of the $S$-module $S f$, i.e., an element of the form $\partial\lrcorner f$ for $\partial \in S$. By definition, these elements form an $S$-submodule of $P$, in particular a $k$-linear subspace.

Let $A=S / I$ be an Artin quotient of $S$; then $A$ is local. The contraction action associates to $A$ an $S$-submodule $M \subseteq P$ consisting of elements annihilated by $I$, so that $A$ and $M$ are dual. If $A$ is Gorenstein, then the $S$-module $M$ is cyclic, generated by a polynomial $f$ of degree $s$ equal to the socle degree of $A$. We call every such $f$ a dual socle generator of the Artin Gorenstein algebra A. Unlike $M$, the polynomial $f$ is not determined uniquely by the choice of the presentation $A=S / I$, however, if $f$ and $g$ are two dual socle generators, then $g=\partial\lrcorner f$, where $\partial \in S$ is invertible.

Conversely, let $f \in P$ be a polynomial of degree $s$. We can associate to it the ideal $I:=\operatorname{ann}_{S}(f)$ such that $A:=S / I$ is a local Artin Gorenstein algebra of socle degree $s$. We call $I$ the apolar ideal of $f$ and $A$ the apolar algebra of $f$, which we denote as

$$
A=\operatorname{Apolar}(f) .
$$

From the discussion above it follows that every local Artin Gorenstein algebra is an apolar algebra of some polynomial.

Remark 2.2. Recall that we may think of $S / \mathfrak{m}_{S}^{s+1}$ as the linear space dual to $P_{\leq s}$. An automorphism $\psi$ of $S$ or $S / \mathfrak{m}_{S}^{s+1}$ induces an automorphism $\psi^{*}$ of the $k$-linear space $P_{\leq s}$. If $f \in P_{\leq s}$ and $I$ is the apolar ideal of $f$, then $\psi(I)$ is the apolar ideal of $\psi^{*}(f)$. Moreover, $f$ and $\psi^{*}(f)$ have the same degree.

2C. Iarrobino's symmetric decomposition of Hilbert function. One of the most important invariants possessed by a local Artin Gorenstein algebra is the symmetric decomposition of its Hilbert function, due to Iarrobino [1994]. To state the theorem it is convenient to define addition of vectors of different lengths position-wise: if $a=\left(a_{0}, \ldots, a_{n}\right)$ and $b=\left(b_{0}, \ldots, b_{m}\right)$ are vectors, then $a+b=$ $\left(a_{0}+b_{0}, \ldots, a_{\max (m, n)}+b_{\max (m, n)}\right)$, where $a_{i}=0$ for $i>n$ and $b_{i}=0$ for $i>m$. In the following, all vectors are indexed starting from zero.

Let $(A, \mathfrak{m}, k)$ be a local Artin Gorenstein algebra. By $\left(0: \mathfrak{m}^{l}\right)$ we denote the annihilator of $\mathfrak{m}^{l}$ in $A$. The chain $0=\left(0: \mathfrak{m}^{0}\right) \subseteq\left(0: \mathfrak{m}^{1}\right) \subseteq \cdots$ defines a filtration on $A$. In general, it is different from the usual filtration $0=\mathfrak{m}^{s+1} \subseteq \mathfrak{m}^{s} \subseteq \mathfrak{m}^{s-1} \subseteq \cdots$. The analysis of mutual position of these filtrations is the content of Theorem 2.3.

Theorem 2.3 (Iarrobino's symmetric decomposition of the Hilbert function). Let $(A, \mathfrak{m}, k)$ be a local Artin Gorenstein algebra of socle degree $s$ with Hilbert function $\mathrm{H}_{A}$. Let

$$
\Delta_{i}(t):=\operatorname{dim}_{k} \frac{\left(0: \mathfrak{m}^{s+1-i-t}\right) \cap \mathfrak{m}^{t}}{\left(0: \mathfrak{m}^{s-i-t}\right) \cap \mathfrak{m}^{t}+\left(0: \mathfrak{m}^{s+1-i-t}\right) \cap \mathfrak{m}^{t+1}} \quad \text { for } t=0,1, \ldots, s-i .
$$


The vectors $\Delta_{0}, \Delta_{1}, \ldots, \Delta_{s}$ have the following properties:

(1) The vector $\Delta_{i}$ has length $s+1-i$ and satisfies $\Delta_{i}(t)=\Delta_{i}(s-i-t)$ for all integers $t \in[0, s-i]$.

(2) The Hilbert function $H_{A}$ is equal to the sum $\sum_{i=0}^{s} \Delta_{i}$.

(3) The vector $\Delta_{0}$ is equal to the Hilbert function of a local Artin Gorenstein graded algebra of socle degree $s$.

Let $(A, \mathfrak{m}, k)$ be a local Artin Gorenstein algebra. There are a few important remarks to make.

(1) Since $\Delta_{0}$ is the Hilbert function of an algebra, we have $\Delta_{0}(0)=1=H_{A}(0)$. Thus, for every $i>0$ we have $\Delta_{i}(0)=0$. From symmetry, it follows that $\Delta_{i}(s+1-i)=0$. In particular, $\Delta_{s}=(0)$ and $\Delta_{s-1}=(0,0)$, so we may ignore these vectors. On the other hand, $\Delta_{s-2}=(0, q, 0)$ is in general nonzero and its importance is illustrated by Proposition 4.5.

(2) Suppose that $H_{A}=(1, n, 1,1)$ for some $n>0$. Then we have $\Delta_{0}=(1, *, *, 1)$ and $\Delta_{1}=(0, *, 0)$, thus $\Delta_{0}=(1, *, 1,1)$, so that $\Delta_{0}=(1,1,1,1)$ because of its symmetry. Then $\Delta_{1}=(0, n-1,0)$. Similarly, if $H_{A}=(1, n, e, 1)$ is the Hilbert function of a local Artin Gorenstein algebra, then $n \geq e$. This is a basic example of how Theorem 2.3 imposes restrictions on the Hilbert function of $A$.

(3) If $A$ is graded, then $\Delta_{0}=H_{A}$ and all other $\Delta_{\text {. }}$ are zero vectors; see [Iarrobino 1994, Proposition 1.7].

(4) For every $a \leq s$ the partial sum $\sum_{i=0}^{a} \Delta_{i}$ is the Hilbert function of a local Artin graded algebra; see [Iarrobino 1994, Definition 1.3, Theorem 1.5]; see also [Iarrobino 1994, Subsection 1.F]. In particular, it satisfies Macaulay's growth theorem; see Section 2E. Thus, e.g., there is no local Artin Gorenstein algebra with Hilbert function decomposition satisfying $\Delta_{0}=(1,1,1,1,1,1)$ and $\Delta_{1}=(0,0,1,0,0)$, because then $\left(\Delta_{0}+\Delta_{1}\right)(1)=1$ and $\left(\Delta_{0}+\Delta_{1}\right)(2)=2$.

Let us now analyze the case when $A=\operatorname{Apolar}(f)=S / \operatorname{ann}_{S}(f)$ is the apolar algebra of a polynomial $f \in P$, where $f=\sum_{i=0}^{s} f_{i}$ for some $f_{i} \in P_{i}$. Each local Artin Gorenstein algebra is isomorphic to such an algebra; see Section 2B. For the proofs of the following remarks, see [Iarrobino 1994].

(1) The vector $\Delta_{0}$ is equal to the Hilbert function of Apolar $\left(f_{s}\right)$, the apolar algebra of the leading form of $f$.

(2) If $A$ is graded, then $\operatorname{ann}_{S}(f)=\operatorname{ann}_{S}\left(f_{S}\right)$, so that we may always assume that $f=f_{s}$. Moreover, in this case $H_{A}(m)$ is equal to $\left(S f_{s}\right)_{m}$, the number of degree- $m$ derivatives of $f_{s}$.

(3) Let $f_{1}$ and $f_{2}$ be polynomials of degree $s$ such that $f_{1}-f_{2}$ is a polynomial of degree $d<s$. Let $A_{i}=\operatorname{Apolar}\left(f_{i}\right)$ and let $\Delta_{A_{i}, m}$ be the symmetric decomposition of 
the Hilbert function $H_{A_{i}}$ of $A_{i}$ for $i=1,2$. Then $\Delta_{A_{1}, m}=\Delta_{A_{2}, m}$ for all $m<s-d$; see [Iarrobino 1994, Lemma 1.10].

2D. Smoothability and unobstructedness. An Artin algebra $A$ is called smoothable if it is a (finite flat) limit of smooth algebras, i.e., if there exists a finite flat family over an irreducible base with a special fiber isomorphic to Spec $A$ and general fiber smooth. Recall that $A \simeq A_{\mathfrak{m}_{1}} \times \cdots \times A_{\mathfrak{m}_{r}}$, where the $\mathfrak{m}_{i}$ are maximal ideals of $A$. The algebra $A$ is smoothable if all localizations $A_{\mathfrak{m}}$ at its maximal ideals are smoothable. The converse also holds, that is, if an algebra $A \simeq B_{1} \times B_{2}$ is smoothable, then the algebras $B_{1}$ and $B_{2}$ are also smoothable; a complete and characteristic-free proof of this fact will appear shortly in [Buczyński and Jelisiejew 2014]. We say that a zero-dimensional scheme $Z=\operatorname{Spec} A$ is smoothable if the algebra $A$ is smoothable.

It is crucial that every local Artin Gorenstein algebra $A$ with $H_{A}(1) \leq 3$ is smoothable — see [Casnati and Notari 2009, Proposition 2.5] — which follows from the Buchsbaum-Eisenbud [1977] classification of resolutions. Also, complete intersections are smoothable. A complete intersection $Z \subseteq \mathbb{P}^{n}$ is smoothable by Bertini's theorem (see [Hartshorne 2010, Example 29.0.1], but note that Hartshorne uses a slightly weaker definition of smoothability, without a finiteness assumption). If $Z=\operatorname{Spec} A$ is a complete intersection in $\mathbb{A}^{n}$, then $Z$ is a union of connected components of a complete intersection $Z^{\prime}=\operatorname{Spec} B$ in $\mathbb{P}^{n}$, so that $B \simeq A \times C$ for some algebra $C$. The algebra $B$ is smoothable since $Z^{\prime}$ is. Thus also the algebra $A$ is smoothable, i.e., $Z$ is smoothable.

Definition 2.4. A smoothable Artin algebra $A$ of length $d$, corresponding to the subset $\operatorname{Spec} A$ of $\mathbb{P}^{n}$, is unobstructed if the tangent space to $\mathcal{H i l b}_{d}\left(\mathbb{P}^{n}\right)$ at the $k$-point [Spec $A]=: p$ has dimension $n d$. If $A$ is unobstructed, then $p$ is a smooth point of the Hilbert scheme.

The unobstructedness is independent of $n$ and the chosen embedding of Spec $A$ into $\mathbb{P}^{n}$; see the discussion before [Casnati and Notari 2009, Lemma 2.3]. The argument above shows that algebras corresponding to complete intersections in $\mathbb{A}^{n}$ and $\mathbb{P}^{n}$ are unobstructed. Every local Artin Gorenstein algebra $A$ with $H_{A}(1) \leq 3$ is unobstructed; see [Casnati and Notari 2009, Proposition 2.5]. Moreover, every local Artin Gorenstein algebra $A$ with $H_{A}(1) \leq 2$ is a complete intersection in $\mathbb{A}^{2}$, by the Hilbert-Burch theorem.

Definition 2.5. An Artin algebra $A$ is limit-reducible if there exists a flat family (over an irreducible base) whose special fiber is $A$ and general fiber is reducible. An Artin algebra $A$ is strongly nonsmoothable if it is not limit-reducible.

Clearly, strongly nonsmoothable algebras (other than $A=k$ ) are nonsmoothable. The definition of strong nonsmoothability is useful, because to show that there is 
no nonsmoothable algebra of length less than $d$ it is enough to show that there is no strongly nonsmoothable algebra of length less than $d$.

2E. Macaulay's growth theorem. We will recall Macaulay's growth theorem and Gotzmann's persistence theorem, which provide strong restrictions on the possible Hilbert functions of graded algebras. Fix $n \geq 1$. Let $m$ be any natural number; then $m$ may be uniquely written in the form

$$
m=\left(\begin{array}{c}
m_{n} \\
n
\end{array}\right)+\left(\begin{array}{c}
m_{n-1} \\
n-1
\end{array}\right)+\cdots+\left(\begin{array}{c}
m_{1} \\
1
\end{array}\right),
$$

where $m_{n}>m_{n-1}>\cdots>m_{1}$. We define

$$
m^{\langle i\rangle}:=\left(\begin{array}{c}
m_{n}+1 \\
n+1
\end{array}\right)+\left(\begin{array}{c}
m_{n-1}+1 \\
n
\end{array}\right)+\cdots+\left(\begin{array}{c}
m_{1}+1 \\
2
\end{array}\right) .
$$

It is useful to compute some initial values of the above-defined function: $1^{\langle n\rangle}=1$ for all $n, 3^{\langle 2\rangle}=4,4^{\langle 2\rangle}=5,6^{\langle 2\rangle}=10$ and $4^{\langle 3\rangle}=5$.

Theorem 2.6 (Macaulay's growth theorem). If A is a graded quotient of a polynomial ring over $k$, then the Hilbert function $H_{A}$ of A satisfies $H_{A}(m+1) \leq H_{A}(m)^{\langle m\rangle}$ for all $\mathrm{m}$.

Proof. See [Bruns and Herzog 1993, Theorem 4.2.10].

Note that the assumptions of Theorem 2.6 are satisfied for every local Artin $k$-algebra $(A, \mathfrak{m}, k)$, since its Hilbert function is by definition equal to the Hilbert function of the associated graded algebra.

Remark 2.7. We will frequently use this easy consequence of Theorem 2.6:

Let $A$ be a graded quotient of a polynomial ring over $k$. Suppose that $H_{A}(l) \leq l$ for some $l$. Then $H_{A}(l)=\left(\begin{array}{l}l \\ l\end{array}\right)+\left(\begin{array}{c}l-1 \\ l-1\end{array}\right)+\cdots$ and $H_{A}(l)^{\langle l\rangle}=\left(\begin{array}{l}l+1 \\ l+1\end{array}\right)+\left(\begin{array}{l}l \\ l\end{array}\right)+\cdots=H_{A}(l)$, thus $H_{A}(l+1) \leq H_{A}(l)$. It follows that the Hilbert function of $H_{A}$ satisfies $H_{A}(l) \geq H_{A}(l+1) \geq H_{A}(l+2) \geq \cdots$. In particular, $H_{A}(m) \leq l$ for all $m \geq l$.

Theorem 2.8 (Gotzmann's persistence theorem). Let $A=S_{\text {poly }} / I$ be a graded quotient of a polynomial ring $S_{\text {poly }}$ over $k$ and suppose that for some $l$ we have $H_{A}(l+1)=H_{A}(l)^{\langle l\rangle}$ and that $I$ is generated by elements of degree at most $l$. Then $H_{A}(m+1)=H_{A}(m)^{\langle m\rangle}$ for all $m \geq l$.

Proof. See [Bruns and Herzog 1993, Theorem 4.3.3].

We will mostly use the following consequence of Theorem 2.8, for which we introduce some (nonstandard) notation. Let $I \subseteq S_{\text {poly }}=k\left[\alpha_{1}, \ldots, \alpha_{n}\right]$ be a graded ideal in a polynomial ring and $m \geq 0$. We say that $I$ is $m$-saturated if, for all $l \leq m$ and $\sigma \in\left(S_{\text {poly }}\right)_{l}$, the condition $\sigma \cdot\left(\alpha_{1}, \ldots, \alpha_{n}\right)^{m-l} \subseteq I$ implies $\sigma \in I$. 
Lemma 2.9. Let $S_{\text {poly }}=k\left[\alpha_{1}, \ldots, \alpha_{n}\right]$ be a polynomial ring with maximal ideal $\mathfrak{n}=\left(\alpha_{1}, \ldots, \alpha_{n}\right)$. Let $I \subseteq S_{\text {poly }}$ be a graded ideal and $A=S_{\text {poly }} / I$. Suppose that $I$ is $m$-saturated for some $m \geq 2$. Then:

(1) If $H_{A}(m)=m+1$ and $H_{A}(m+1)=m+2$, then $H_{A}(l)=l+1$ for all $l \leq m$; in particular, $H_{A}(1)=2$.

(2) If $H_{A}(m)=m+2$ and $H_{A}(m+1)=m+3$, then $H_{A}(l)=l+2$ for all $l \leq m$; in particular, $H_{A}(1)=3$.

Proof. (1) First, if $H_{A}(l) \leq l$ for some $l<m$ then, by Macaulay's growth theorem, $H_{A}(m) \leq l<m+1$, a contradiction. So it suffices to prove that $H_{A}(l) \leq l+1$ for all $l<m$.

Let $J$ be the ideal generated by elements of degree at most $m$ in $I$. We will prove that the graded ideal $J$ of $S_{\text {poly }}$ defines a $\mathbb{P}^{1}$ linearly embedded into $\mathbb{P}^{n-1}$.

Let $B=S_{\text {poly }} / J$. Then $H_{B}(m)=m+1$ and $H_{B}(m+1) \geq m+2$. Since $H_{B}(m)=m+1=\left(\begin{array}{c}m+1 \\ m\end{array}\right)$, we have $H_{B}(m)^{\langle m\rangle}=\left(\begin{array}{c}m+2 \\ m+1\end{array}\right)=m+2$ and, by Theorem 2.6, we get $H_{B}(m+1) \leq m+2$, thus $H_{B}(m+1)=m+2$. Then, by Gotzmann's persistence theorem, $H_{B}(l)=l+1$ for all $l>m$. This implies that the Hilbert polynomial of Proj $B \subseteq \mathbb{P}^{n-1}$ is $h_{B}(t)=t+1$, so that Proj $B \subseteq \mathbb{P}^{n-1}$ is a linearly embedded $\mathbb{P}^{1}$. In particular, the Hilbert function and Hilbert polynomial of Proj $B$ are equal for all arguments. By assumption, we have $J_{l}=J_{l}^{\text {sat }}$ for all $l<m$. Then $H_{A}(l)=H_{S_{\text {poly }} / J}(l)=H_{S_{\text {poly }} / J} J^{\text {sat }}(l)=l+1$ for all $l<m$ and the claim of the lemma follows.

(2) The proof is similar to that of (1); we mention only the points where it changes. Let $J$ be the ideal generated by elements of degree at most $m$ in $I$ and $B=S_{\text {poly }} / J$. Then $H_{B}(m)=m+2=\left(\begin{array}{c}m+1 \\ m\end{array}\right)+\left(\begin{array}{c}m-1 \\ m-1\end{array}\right)$, thus $H_{B}(m+1) \leq\left(\begin{array}{c}m+2 \\ m+1\end{array}\right)+\left(\begin{array}{c}m \\ m\end{array}\right)=m+3$ and $B$ defines a closed subscheme of $\mathbb{P}^{n-1}$ with Hilbert polynomial $h_{B}(t)=t+2$. There are two isomorphism types of such subschemes: the union of $\mathbb{P}^{1}$ and a point, and $\mathbb{P}^{1}$ with an embedded double point. One checks that for these schemes the Hilbert polynomial is equal to the Hilbert function for all arguments and then proceeds as in the proof of part (1).

Remark 2.10. If $A=S_{\text {poly }} / I$ is a graded Artin Gorenstein algebra of socle degree $s$, then it is $m$-saturated for every $m \leq s$. Indeed, we may assume that $A=\operatorname{Apolar}(F)$ for some homogeneous $F \in P$ of degree $s$; then $I=\operatorname{ann}_{S}(F)$. Let $\mathfrak{n}=\left(\alpha_{1}, \ldots, \alpha_{n}\right) \subseteq k\left[\alpha_{1}, \ldots, \alpha_{n}\right]=S_{\text {poly }}$. Take $\sigma \in\left(S_{\text {poly }}\right)_{l}$; then $\sigma \in I$ if and only if $\sigma\lrcorner F=0$. Similarly, $\sigma \mathfrak{n}^{m-l} \subseteq I$ if and only if every element of $\mathfrak{n}^{m-l}$ annihilates $\sigma\lrcorner F$. Since $\sigma\lrcorner F$ is either a homogeneous polynomial of degree $s-l \geq m-l$ or it is zero, both conditions are equivalent.

Remark 2.11. Clearly, if two graded ideals $I$ and $J$ of $S_{\text {poly }}$ agree up to degree $m$ and $I$ is $m$-saturated, then also $J$ is $m$-saturated. 
2F. Flatness over Spec $k[t]$. For further reference we explicitly state a purely elementary flatness criterion. Its formulation is a bit complicated, but this is precisely the form which is needed for the proofs. This criterion relies on the easy observation that the torsion-free modules over $k[t]$ are flat.

Proposition 2.12. Suppose $S$ is a $k$-module and $I \subseteq S[t]$ is a $k[t]$-submodule. Let $I_{0}:=I \cap S$. If, for every $\lambda \in k$, we have

$$
(t-\lambda) \cap I \subseteq(t-\lambda) I+I_{0}[t],
$$

then $S[t] / I$ is a flat $k[t]$-module.

Proof. The ring $k[t]$ is a principal ideal domain; thus, a $k[t]$-module is flat if and only if it is torsion-free; see [Eisenbud 1995, Corollary 6.3]. Since every polynomial in $k[t]$ decomposes into linear factors, to prove that $M=S[t] / I$ is torsion-free it is enough to show that $t-\lambda$ are non-zerodivisors on $M$, i.e., that $(t-\lambda) x \in I$ implies $x \in I$ for all $x \in S[t], \lambda \in k$.

Fix $\lambda \in k$ and suppose that $x \in S[t]$ is such that $(t-\lambda) x \in I$. Then, by assumption, $(t-\lambda) x \in(t-\lambda) I+I_{0}[t]$, so that $(t-\lambda)(x-i) \in I_{0}[t]$ for some $i \in I$. Since $S[t] / I_{0}[t] \simeq S / I_{0}[t]$ is a free $k[t]$-module, we have $x-i \in I_{0}[t] \subseteq I$ and so $x \in I$.

Remark 2.13. Let $i_{1}, \ldots, i_{r}$ be the generators of $I$. To check the inclusion which is the assumption of Proposition 2.12, it is enough to check that $s \in(t-\lambda) \cap I$ implies $s \in(t-\lambda) I+I_{0}[t]$ for all $s=s_{1} i_{1}+\cdots+s_{r} i_{r}$, where $s_{i} \in S$.

Indeed, take an arbitrary element $s \in I$ and write $s=t_{1} i_{1}+\cdots+t_{r} i_{r}$, where $t_{1}, \ldots, t_{r} \in S[t]$. Dividing $t_{i}$ by $t-\lambda$, we obtain $s=s_{1} i_{1}+\cdots+s_{r} i_{r}+(t-\lambda) i$, where $i \in I$ and $s_{i} \in S$. Let $s^{\prime}=s_{1} i_{1}+\cdots+s_{r} i_{r}$; then $s \in(t-\lambda) \cap I$ if and only if $s^{\prime} \in(t-\lambda) \cap I$, and $s \in(t-\lambda) I+I_{0}[t]$ if and only if $s^{\prime} \in(t-\lambda) I+I_{0}[t]$.

Example 2.14. Consider $S=k[x, y]$ and $I=x y S[t]+\left(x^{3}-t x\right) S[t] \subseteq S[t]$. Take an element $s_{1} x y+s_{2}\left(x^{3}-t x\right) \in I$ and suppose $s_{1} x y+s_{2}\left(x^{3}-t x\right) \in(t-\lambda) S[t]$. We want to prove that this element lies in $I_{0}[t]+(t-\lambda) I$. As in Remark 2.13, by subtracting an element of $I(t-\lambda)$ we may assume that $s_{1}$ and $s_{2}$ lie in $S$. Then $s_{1} x y+s_{2}\left(x^{3}-t x\right) \in(t-\lambda) S[t]$ if and only if $s_{1} x y+s_{2}\left(x^{3}-\lambda x\right)=0$. In particular, we have $s_{2} \in y S$, so that $s_{2}\left(x^{3}-t x\right) \in x y S[t]$; then $s_{1} x y+s_{2}\left(x^{3}-t x\right) \in x y S[t] \subseteq I_{0}[t]$.

Similarly, we will frequently use the following easy observation:

Lemma 2.15. Consider a ring $R=B[\alpha]$ graded by the degree of $\alpha$. Let $d$ be a natural number and $I \subseteq R$ be a homogeneous ideal generated in degrees less or equal to $d$.

Let $q \in B[\alpha]$ be an element of $\alpha$-degree strictly less than d such that for every $b \in B$ satisfying $b \alpha^{d} \in I$, we have $b q \in I$. Then, for every $r \in R$,

$$
r\left(\alpha^{d}-q\right) \in I \Rightarrow r \alpha^{d} \in I \quad \text { and } \quad r q \in I .
$$


Proof. We apply induction on $\alpha$-degree of $r$, the base case being $r=0$. Write

$$
r=\sum_{i=0}^{m} r_{i} \alpha^{i}, \quad \text { where } \quad r_{i} \in B .
$$

The leading form of $r\left(\alpha^{d}-q\right)$ is $r_{m} \alpha^{m+d}$ and it lies in $I$. Since $I$ is homogeneous and generated in degree at most $d$, we have $r_{m} \alpha^{d} \in I$. Then $r_{m} q \in I$ by assumption, so that $\hat{r}:=r-r_{m} \alpha^{m}$ satisfies $\hat{r}\left(\alpha^{d}-q\right) \in I$. By induction we have $\hat{r} \alpha^{d}, \hat{r} q \in I$, then also $r \alpha^{d}, r q \in I$.

\section{Standard form of the dual generator}

Definition 3.1. Let $f \in P=k\left[x_{1}, \ldots, x_{n}\right]$ be a polynomial of degree $s$. Let $I=\operatorname{ann}_{S}(f)$ and $A=S / I=$ Apolar $(f)$. By $\Delta$. we denote the decomposition of the Hilbert function of $A$ and we set $e(a):=\sum_{t=0}^{a} \Delta_{t}(1)$.

We say that $f$ is in the standard form if $f=f_{0}+f_{1}+f_{2}+f_{3}+\cdots+f_{s}, \quad$ where $f_{i} \in P_{i} \cap k\left[x_{1}, \ldots, x_{e(s-i)}\right]$ for all $i$.

Note that if $f$ is in the standard form and $\partial \in \mathfrak{m}_{S}$, then $\left.f+\partial\right\lrcorner f$ is also in the standard form. We say that an Artin Gorenstein algebra $S / I$ is in the standard form if any (or every) dual socle generator of $S / I$ is in the standard form; see Proposition 3.5 below.

Example 3.2. If $f=x_{1}^{6}+x_{2}^{5}+x_{3}^{3}$, then $f$ is in the standard form. Indeed, $e(0)=1$, $e(1)=2, e(2)=2, e(3)=3$ so that we should check that $x_{1}^{6} \in k\left[x_{1}\right], x_{2}^{5} \in k\left[x_{1}, x_{2}\right]$ and $x_{3}^{3} \in k\left[x_{1}, x_{2}, x_{3}\right]$, which is true. To contrast, $g=x_{3}^{6}+x_{2}^{5}+x_{1}^{3}$ is not in the standard form, but may be put in the standard form via a change of variables.

The change of variables procedure of Example 3.2 may be generalized to prove that every local Artin Gorenstein algebra can be put in a standard form:

Proposition 3.3. For every Artin Gorenstein algebra $S / I$ there is an automorphism $\varphi: S \rightarrow S$ such that $S / \varphi(I)$ is in the standard form.

Proof. See [Iarrobino 1994, Theorem 5.3AB]; the proof is rewritten in [Jelisiejew 2014a, Theorem 4.38].

The idea of the proof of Proposition 3.3 is to "linearize" some elements of $S$. This is quite technical and perhaps it can be best seen in the following example.

Example 3.4. Let $f=x_{1}^{6}+x_{1}^{4} x_{2}$. The annihilator of $f$ in $S$ is $\left(\alpha_{2}^{2}, \alpha_{1}^{5}-\alpha_{1}^{3} \alpha_{2}\right)$, the Hilbert function of Apolar $(f)$ is $(1,2,2,2,1,1,1)$ and the symmetric decomposition is

$$
\Delta_{0}=(1,1,1,1,1,1,1), \quad \Delta_{1}=(0,0,0,0,0,0), \quad \Delta_{2}=(0,1,1,1,0) .
$$


This shows that $e(0)=1, e(1)=1$ and $e(2)=2$. If $f$ is in the standard form we should have $f_{5}=x_{1}^{4} x_{2} \in k\left[x_{1}, \ldots, x_{e(1)}\right]=k\left[x_{1}\right]$. This means that $f$ is not in the standard form. The "reason" for $e(1)=1$ is the fact that $\alpha_{1}^{3}\left(\alpha_{2}-\alpha_{1}^{2}\right)$ annihilates $f$, and the "reason" for $f_{5} \notin k\left[x_{1}\right]$ is that $\alpha_{2}-\alpha_{1}^{2}$ is not a linear form. Thus we make $\alpha_{2}-\alpha_{1}^{2}$ a linear form by twisting by a suitable automorphism of $S$.

We define an automorphism $\psi: S \rightarrow S$ by $\psi\left(\alpha_{1}\right)=\alpha_{1}$ and $\psi\left(\alpha_{2}\right)=\alpha_{2}+\alpha_{1}^{2}$, so that we have $\psi\left(\alpha_{2}-\alpha_{1}^{2}\right)=\alpha_{2}$. The automorphism maps the annihilator of $f$ to the ideal $I:=\left(\left(\alpha_{2}+\alpha_{1}^{2}\right)^{2}, \alpha_{1}^{3} \alpha_{2}\right)$. We will see that the algebra $S / I$ is in the standard form and also find a particular dual generator obtained from $f$.

As mentioned in Remark 2.2, the automorphism $\psi$ induces an automorphism $\psi^{*}$ of the $k$-linear space $P_{\leq 6}$. This automorphism maps $f$ to a dual socle generator $\psi^{*} f$ of $S / I$.

The element $F:=\psi^{*} x_{1}^{6}$ is the only element of $P$ such that $\left.\psi\left(\alpha_{1}^{7}\right)\right\lrcorner F=$ $\left.\psi\left(\alpha_{2}\right)\right\lrcorner F=0, \psi\left(\alpha_{1}^{6}\right)(F)=1$ and $\psi\left(\alpha_{1}^{l}\right)(F)=0$ for $l \leq 5$. Caution: in the last line we use evaluation on the functional and not the induced action (see Remark 2.2). One can compute that $\psi^{*} x_{1}^{6}=x_{1}^{6}-x_{1}^{4} x_{2}+x_{1}^{2} x_{2}^{2}-x_{2}^{3}$ and similarly $\psi^{*} x_{1}^{4} x_{2}=x_{1}^{4} x_{2}-2 x_{1}^{2} x_{2}+3 x_{2}^{3}$, so that $\psi^{*} f=x_{1}^{6}-x_{1}^{2} x_{2}^{2}+2 x_{2}^{3}$. Now indeed $x_{1}^{6} \in k\left[x_{1}\right], x_{1}^{2} x_{2}^{2} \in k\left[x_{1}, x_{2}\right]$ and $2 x_{2}^{3} \in k\left[x_{1}, x_{2}\right]$, so the dual socle generator is in the standard form.

We note the following equivalent conditions for a dual socle generator to be in the standard form:

Proposition 3.5. In the notation of Definition 3.1, the following conditions are equivalent for a polynomial $f \in P$ :

(1) The polynomial $f$ is in the standard form.

(2) For all $r$ and $i$ such that $r>e(s-i)$, we have $\mathfrak{m}_{S}^{i-1} \alpha_{r} \subseteq I=(f)^{\perp}$. Equivalently, for all $r$ and $i$ such that $r>e(i)$, we have $\mathfrak{m}_{S}^{s-i-1} \alpha_{r} \subseteq I=(f)^{\perp}$.

Proof. Straightforward.

Corollary 3.6. Let $f \in P$ be such that the algebra $S / I$ is in the standard form, where $I=\operatorname{ann}_{S}(f)$. Let $\varphi$ be an automorphism of $S$ given by

$$
\varphi\left(\alpha_{i}\right)=\kappa_{i} \alpha_{i}+q_{i},
$$

where $q_{i}$ is such that $\left.\left.\operatorname{deg}\left(q_{i}\right\lrcorner f\right) \leq \operatorname{deg}\left(\alpha_{i}\right\lrcorner f\right)$ and $\kappa_{i} \in k \backslash\{0\}$. Then the algebra $S / \varphi^{-1}(I)$ is also in the standard form.

Proof. The algebras $S / I$ and $S / \varphi^{-1}(I)$ are isomorphic; in particular, they have equal functions $e(\cdot)$. By Proposition 3.5 it suffices to prove that if, for some $r$ and $i$, we have $\mathfrak{m}_{S}^{r} \alpha_{i} \subseteq I$, then $\mathfrak{m}_{S}^{r} \alpha_{i} \subseteq \varphi^{-1}(I)$. The latter condition is equivalent to $\mathfrak{m}_{S}^{r} \varphi\left(\alpha_{i}\right) \subseteq I$. If $\left.\mathfrak{m}_{S}^{r} \alpha_{i}\right\lrcorner f=0$ then $\left.\operatorname{deg}\left(\alpha_{i}\right\lrcorner f\right)<r$ so, by assumption, $\left.\operatorname{deg}\left(q_{i}\right\lrcorner f\right)<r$; thus $\left.\mathfrak{m}_{S}^{r} q_{i}\right\lrcorner f=0$ and $\left.\mathfrak{m}_{S}^{r} \varphi\left(\alpha_{i}\right)=\mathfrak{m}_{S}^{r}\left(\kappa_{i} \alpha_{i}+q_{i}\right)\right\lrcorner f=0$. 
Corollary 3.7. Suppose that $q \in \mathfrak{m}_{S}^{2}$ does not contain $\alpha_{i}$ and let $\varphi: S \rightarrow S$ be an automorphism given by

$$
\varphi\left(\alpha_{j}\right)= \begin{cases}\alpha_{j} & \text { if } j \neq i, \\ \kappa_{i} \alpha_{i}+q & \text { if } j=i,\end{cases}
$$

where $\kappa_{i} \in k \backslash\{0\}$. Suppose that $S / I$ is in the standard form, where $I=\operatorname{ann}_{S}(f)$, and that $\left.\operatorname{deg}(q\lrcorner f) \leq \operatorname{deg}\left(\alpha_{i}\right\lrcorner f\right)$. Then the algebras $S / \varphi(I)$ and $S / \varphi^{-1}(I)$ are also in the standard form.

Proof. Note that $\psi: S \rightarrow S$, given by $\psi\left(\alpha_{j}\right)=\alpha_{j}$ for $j \neq i$ and $\psi\left(\alpha_{i}\right)=\kappa_{i}^{-1}\left(\alpha_{i}-q\right)$, is an automorphism of $S$ and, furthermore, $\psi\left(\kappa_{i} \alpha_{i}+q\right)=\alpha_{i}-q+q=\alpha_{i}$, so that $\psi=\varphi^{-1}$. Both $\varphi$ and $\psi$ satisfy assumptions of Corollary 3.6, so both $S / \varphi^{-1}(I)$ and $S / \psi^{-1}(I)=S / \varphi(I)$ are in the standard form.

Remark 3.8. The assumption $q \in \mathfrak{m}_{S}^{2}$ of Corollary 3.7 is needed only to ensure that $\varphi$ is an automorphism of $S$. On the other hand, the fact that $q$ does not contain $\alpha_{i}$ is important, because it allows us to control $\varphi^{-1}$ and, in particular, prove that $S / \varphi(I)$ is in the standard form.

The following corollary is a straightforward generalization of Corollary 3.7, but the notation is difficult. We first choose a set $\mathcal{K}$ of variables. The automorphism sends each variable from $\mathcal{K}$ to (a multiple of) itself plus a suitable polynomial in variables not appearing in $\mathcal{K}$.

Corollary 3.9. Take $\mathcal{K} \subseteq\{1,2, \ldots, n\}$ and $q_{i} \in \mathfrak{m}_{S}^{2}$ for $i \in \mathcal{K}$ which do not contain any variables from the set $\left\{\alpha_{i}\right\}_{i \in \mathcal{K}}$. Define $\varphi: S \rightarrow S$ by

$$
\varphi\left(\alpha_{i}\right)= \begin{cases}\alpha_{i} & \text { if } i \notin \mathcal{K} \\ \kappa_{i} \alpha_{i}+q_{i} & \text { if } i \in \mathcal{K}\end{cases}
$$

where $\kappa_{i} \in k \backslash\{0\}$. Suppose that $S / I$ is in the standard form, where $I=\operatorname{ann}_{S}(f)$, and that $\left.\left.\operatorname{deg}\left(q_{i}\right\lrcorner f\right) \leq \operatorname{deg}\left(\alpha_{i}\right\lrcorner f\right)$ for all $i \in \mathcal{K}$. Then the algebras $S / \varphi(I)$ and $S / \varphi^{-1}(I)$ are also in the standard form.

\section{Special forms of dual socle generators}

Recall that $k$ is an algebraically closed field of characteristic neither 2 nor 3.

In the previous section we mentioned that for every local Artin Gorenstein algebra there exists a dual socle generator in the standard form; see Definition 3.1. In this section we will see that in most cases we can say more about this generator. Our main aim is to put the generator in the form $x^{s}+f$, where $f$ contains no monomial divisible by a "high" power of $x$. We will use it to prove that families arising from certain ray decompositions (see Definition 5.2) are flat.

We begin with an easy observation. 
Remark 4.1. Suppose that a polynomial $f \in P$ is such that $H_{\text {Apolar }(f)}(1)$ equals the number of variables in $P$. Then any linear form in $P$ is a derivative of $f$. If $\operatorname{deg} f>1$ then the $S$-submodules $S f$ and $S\left(f-f_{1}-f_{0}\right)$ are equal, so analyzing this modules we may assume $f_{1}=f_{0}=0$, i.e., the linear part of $f$ is zero.

Later we use this remark implicitly.

The following lemma provides a method to slightly improve the given dual socle generator. This improvement is the building block of all other results in this section.

Lemma 4.2. Let $f \in P$ be a polynomial of degree $s$ and $A$ be the apolar algebra of $f$. Suppose that $\left.\alpha_{1}^{s}\right\lrcorner f \neq 0$. For every $i$, let $\left.d_{i}:=\operatorname{deg}\left(\alpha_{1} \alpha_{i}\right\lrcorner f\right)+2$.

Then $A$ is isomorphic to the apolar algebra of a polynomial $\hat{f}$ of degree s such that $\left.\alpha_{1}^{s}\right\lrcorner \hat{f}=1$ and $\left.\alpha_{1}^{d_{i}-1} \alpha_{i}\right\lrcorner \hat{f}=0$ for all $i \neq 1$. Moreover, the leading forms of $f$ and $\hat{f}$ are equal up to a nonzero constant. If $f$ is in the standard form, then $\hat{f}$ is also in the standard form.

Proof. By multiplying $f$ by a nonzero constant we may assume that $\left.\alpha_{1}^{s}\right\lrcorner f=1$. Denote $I:=\operatorname{ann}_{S}(f)$. Since $\left.\operatorname{deg}\left(\alpha_{1} \alpha_{i}\right\lrcorner f\right)=d_{i}-2$, the polynomial $\left.\alpha_{1}^{d_{i}-1} \alpha_{i}\right\lrcorner f=$ $\left.\alpha_{1}^{d_{i}-2}\left(\alpha_{1} \alpha_{i}\right\lrcorner f\right)$ is constant; we denote it by $\lambda_{i}$. Then

$$
\left.\left(\alpha_{1}^{d_{i}-1} \alpha_{i}-\lambda_{i} \alpha_{1}^{s}\right)\right\lrcorner f=0, \quad \text { so that } \quad \alpha_{1}^{d_{i}-1}\left(\alpha_{i}-\lambda_{i} \alpha_{1}^{s-d_{i}+1}\right) \in I .
$$

Define an automorphism $\varphi: S \rightarrow S$ by

$$
\varphi\left(\alpha_{i}\right)= \begin{cases}\alpha_{1} & \text { if } i=1 \\ \alpha_{i}-\lambda_{i} \alpha_{1}^{s-d_{i}+1} & \text { if } i \neq 1\end{cases}
$$

then $\alpha_{1}^{d_{i}-1} \alpha_{i} \in \varphi^{-1}(I)$ for all $i>1$. The dual socle generator $\hat{f}$ of the algebra $S / \varphi^{-1}(I)$ has the required form. We can easily check that the graded algebras of $S / \varphi^{-1}(I)$ and $S / I$ are equal; in particular, $\hat{f}$ and $f$ have the same leading form, up to a nonzero constant.

Suppose now that $f$ is in the standard form. Let $i \in\{1, \ldots, n\}$. Then $d_{i}=$ $\left.\left.\operatorname{deg}\left(\alpha_{1} \alpha_{i}\right\lrcorner f\right)+2 \leq \operatorname{deg}\left(\alpha_{i}\right\lrcorner f\right)+1$, so that $\left.\left.\operatorname{deg}\left(\alpha_{1}^{s-d_{i}+1}\right\lrcorner f\right) \leq d_{i}-1 \leq \operatorname{deg}\left(\alpha_{i}\right\lrcorner f\right)$. Since $\varphi$ is an automorphism of $S$, by Remark 3.8 we may apply Corollary 3.9 to $\varphi$. Then $S / \varphi(I)$ is in the standard form, so $\hat{f}$ is in the standard form by definition.

Example 4.3. Let $f \in k\left[x_{1}, x_{2}, x_{3}, x_{4}\right]$ be a polynomial of degree $s$. Suppose that the leading form $f_{s}$ of $f$ can be written as $f_{s}=x_{1}^{s}+g_{s}$, where $g_{s} \in k\left[x_{2}, x_{3}, x_{4}\right]$. Then $\left.\operatorname{deg}\left(\alpha_{1} \alpha_{i}\right\lrcorner f\right) \leq s-3$ for all $i>1$. Using Lemma 4.2, we produce $\hat{f}=x_{1}^{s}+h$ such that the apolar algebras of $f$ and $\hat{f}$ are isomorphic and $\left.\alpha_{1}^{s-2} \alpha_{i}\right\lrcorner h=0$ for all $i \neq 1$. Then $\left.\alpha_{1}^{s-2}\right\lrcorner h=\lambda_{1} x_{1}+\lambda_{2}$, where $\lambda_{i} \in k$ for $i=1$, 2. After adding a suitable derivative to $\hat{f}$, we may assume $\lambda_{1}=\lambda_{2}=0$, that is, $\left.\alpha_{1}^{s-2}\right\lrcorner h=0$.

Example 4.4. Suppose that a local Artin Gorenstein algebra $A$ of socle degree $s$ has Hilbert function equal to $\left(1, H_{1}, H_{2}, \ldots, H_{c}, 1, \ldots, 1\right)$. The standard form of 
the dual socle generator of $A$ is

$$
f=x_{1}^{s}+\kappa_{s-1} x_{1}^{s-1}+\cdots+\kappa_{c+2} x_{1}^{c+2}+g,
$$

where deg $g \leq c+1$ and $\kappa_{\bullet} \in k$. By adding a suitable derivative we may, furthermore, make all $\kappa_{i}=0$ and assume that $\left.\alpha_{1}^{c+1}\right\lrcorner g=0$. Using Lemma 4.2 we may also assume that $\left.\alpha_{1}^{c} \alpha_{j}\right\lrcorner g=0$ for every $j \neq 1$, so we may assume $\left.\alpha_{1}^{c}\right\lrcorner g=0$, arguing as in Example 4.3. This gives a dual socle generator

$$
f=x_{1}^{s}+g,
$$

where deg $g \leq c+1$ and $g$ does not contain monomials divisible by $x_{1}^{c}$.

The following proposition was proved in [Casnati and Notari 2014a] under the assumption that $k$ is algebraically closed of characteristic zero and in [Jelisiejew 2014a, Theorem 5.1] under the assumption that $k=\mathbb{C}$. For completeness we include the proof (with no further assumptions on $k$ other than the ones listed at the beginning of this section).

Proposition 4.5. Let $A$ be Artin local Gorenstein algebra of socle degree $s \geq 2$ such that the Hilbert function decomposition from Theorem 2.3 has $\Delta_{A, s-2}=(0, q, 0)$. Then $A$ is isomorphic to the apolar algebra of a polynomial $f$ such that $f$ is in the standard form and the quadric part $f_{2}$ of $f$ is a sum of $q$ squares of variables not appearing in $f_{\geq 3}$ and a quadric in variables appearing in $f_{\geq 3}$.

Proof. Let us take a standard dual socle generator $f \in P:=k\left[x_{1}, \ldots, x_{n}\right]$ of the algebra $A$. Now we will twist $f$ to obtain the required form of $f_{2}$. We may assume that $H_{\text {Apolar }(f)}(1)=n$.

If $s=2$, then the theorem follows from the fact that the quadric $f$ may be diagonalized. Assume $s \geq 3$. Let $e:=e(s-3)=\sum_{t=0}^{s-3} \Delta_{A, t}(1)$. We have $n=e(s-2)=f+q$, so that $f_{\geq 3} \in k\left[x_{1}, \ldots, x_{e}\right]$ and $f_{2} \in k\left[x_{1}, \ldots, x_{n}\right]$. Note that $f_{\geq 3}$ is also in the standard form, so that every linear form in $x_{1}, \ldots, x_{e}$ is a derivative of $f_{\geq 3}$; see Remark 4.1.

First, we want to assure that $\left.\alpha_{n}^{2}\right\lrcorner f \neq 0$. If $\left.\alpha_{n}\right\lrcorner f \in k\left[x_{1}, \ldots, x_{e}\right]$ then there exists an operator $\partial \in \mathfrak{m}_{S}^{2}$ such that $\left.\left(\alpha_{n}-\partial\right)\right\lrcorner f=0$. This contradicts the fact that $f$ was in the standard form (see the discussion in Example 3.4). So we get that $\left.\alpha_{n}\right\lrcorner f$ contains some $x_{r}$ for $r>e$, i.e., $f$ contains a monomial $x_{r} x_{n}$. A change of variables involving only $x_{r}$ and $x_{n}$ preserves the standard form and gives $\left.\alpha_{n}^{2}\right\lrcorner f \neq 0$.

Applying Lemma 4.2 to $x_{n}$, we see that $f$ may be taken to be in the form $\hat{f}+x_{n}^{2}$, where $\hat{f}$ does not contain $x_{n}$, that is, $\hat{f} \in k\left[x_{1}, \ldots, x_{n-1}\right]$. We repeat the argument for $\hat{f}$.

Example 4.6. If $A$ is an algebra of socle degree 3 , then $H_{A}=(1, n, e, 1)$ for some $n$ and $e$. Moreover, $n \geq e$ and the symmetric decomposition of $H_{A}$ is 
$(1, e, e, 1)+(0, n-e, 0)$. By Proposition 4.5 , we see that $A$ is isomorphic to the apolar algebra of

$$
f+\sum_{e<i \leq n} x_{i}^{2}
$$

where $f \in k\left[x_{1}, \ldots, x_{e}\right]$. This claim was first proved by Elias and Rossi [2012, Theorem 4.1].

4A. Irreducibility for fixed Hilbert function in two variables. Below we analyze local Artin Gorenstein algebras with Hilbert function $(1,2,2, \ldots)$. Such algebras are (in some cases) classified up to isomorphism in [Elias and Valla 2011], but rather than such classification we need to know the geometry of their parameter space, which is analyzed (among other such spaces) in [Iarrobino 1977].

We need the following Proposition 4.7, which is part of folklore. We thank J. Buczyński for explaining the proof.

Let $r \geq 1$ be a natural number. By $\mathcal{H} i l b_{r}$ Spec $S$ we denote the Hilbert scheme of length $r$ subschemes of the power series ring $S$. It is called the punctual Hilbert scheme because, as a set, $\mathcal{H i l b}{ }_{r}$ Spec $S$ is equal to the set of length- $r$ subschemes of $\mathbb{P}^{n}$ supported at a single fixed point.

We recall a classical construction. Let $V$ be a constructible subset of $P_{\leq s}$. Assume that the apolar algebra Apolar $(f)$ has length $r$ for every closed point $f \in V$. Then we may construct the incidence scheme $\{(f, \operatorname{Apolar}(f))\} \rightarrow V$, which is a finite flat family over $V$, and thus we obtain a morphism from $V$ to $\mathcal{H} i l b_{r}$ Spec $S$. See [Jelisiejew 2014a, Proposition 4.39] for details.

Proposition 4.7. Let $\mathcal{R} \subseteq \mathcal{H}$ ilb $r$ Spec $S$ be a constructible subset and $V \subseteq P$ denote the set of all possible dual socle generators of elements of $\mathcal{R}$. If $\mathcal{R}$ is irreducible, then also $V$ is irreducible.

Proof. Below, by $k^{*}$ and $S^{*}$ we denote the sets of invertible elements of $k$ and $S$, respectively.

There is an induced surjective morphism $\varphi$ from $V$ to $\mathcal{R}$, as explained above. By construction, the fiber over $\varphi(f)$ is $\left.S^{*}\right\lrcorner f$. The image $\mathcal{R}$ of $\varphi$ is irreducible, so it is enough to show the existence of an open cover $\left\{H_{i}\right\}$ of $\mathcal{R}$ such that every $\varphi^{-1}\left(H_{i}\right)$ is irreducible.

Choose an element $f \in V$ and a section of $\mathfrak{m}_{S} / \operatorname{ann}_{S}(f)$ to $\mathfrak{m}_{S}$, that is, a linear subspace $\mathfrak{m}(f) \subseteq \mathfrak{m}_{S}$ such that $\mathfrak{m}(f) \rightarrow \mathfrak{m}_{S} / \operatorname{ann}_{S}(f)$ is bijective. Let $O(f):=\mathfrak{m}(f)+k \subseteq S$; then $S\lrcorner f=O(f)\lrcorner f$. Finally, let $O(f)^{*}:=k^{*}+\mathfrak{m}(f)$, so that $\left.\varphi^{-1}(\varphi(f))=O(f)^{*}\right\lrcorner f$. Consider the set

$$
\left.\left.U_{f}=\left\{g \in V \mid O(f) \cap \operatorname{ann}_{S}(g)=0\right\}=\{g \in V \mid O(f)\lrcorner g=S\right\lrcorner g\right\} .
$$


It is an open set in $V$ and its image $H_{f}=\varphi\left(U_{f}\right)$ is open (hence irreducible) in the Hilbert scheme. Moreover, $U_{f}=\varphi^{-1}\left(H_{f}\right)$. For every $g \in U_{f}$ the fiber $\varphi^{-1}(\varphi(g))$ is equal to $\left.O(f)^{*}\right\lrcorner g$.

By [Emsalem 1978, Proposition 18 and its Corollary] there is an open neighborhood $H_{f}^{\prime} \subseteq H_{f}$ of $\varphi(f)$ such that the morphism $\varphi: \varphi^{-1}\left(H_{f}^{\prime}\right) \rightarrow H_{f}^{\prime}$ has a section $i$. Denoting $\varphi^{-1}\left(H_{f}^{\prime}\right)$ by $U_{f}^{\prime}$, we have a surjective morphism $O(f)^{*} \times H_{f}^{\prime} \rightarrow U_{f}^{\prime}$ mapping $(\sigma, h)$ to $\sigma\lrcorner i(h)$. Since $O(f)^{*}$ and $H_{f}^{\prime}$ are irreducible, also $U_{f}^{\prime}$ is irreducible. Therefore, $\left\{H_{f}^{\prime}\right\}$ forms a desired cover of $\mathcal{R}$ and so $V$ is irreducible.

Proposition 4.8. Let $H=(1,2,2, *, \ldots, *, 1)$ be a vector of length $s+1$. The set of polynomials $f \in k\left[x_{1}, x_{2}\right]$ such that $H_{\mathrm{Apolar}(f)}=H$ constitutes an irreducible subscheme of the affine space $k\left[x_{1}, x_{2}\right]_{\leq s}$. A general member of this set has, up to an automorphism of $P$ induced by an automorphism of $S$, the form $f+\partial\lrcorner f$, where $f=x_{1}^{s}+x_{2}^{s_{2}}$ for some $s_{2} \leq s$.

Proof. Let $V \subseteq k\left[x_{1}, x_{2}\right]$ denote the set of those $f$ such that $H_{\text {Apolar }(f)}=H$. Then the image of $V$ under the mapping sending $f$ to $\operatorname{Apolar}(f)$ is irreducible by [Iarrobino 1977, Theorem 3.13]. By Proposition 4.7, the set $V$ is irreducible.

In the case $H=(1,1,1, \ldots, 1)$ the claim (with $s_{2}=0$ ) follows directly from the existence of the standard form of a polynomial. Further in the proof, we assume $H(1)=2$.

Let us take a general polynomial $f$ such that $H_{\text {Apolar }(f)}=H$. Then $\operatorname{ann}_{S}(f)=$ $\left(q_{1}, q_{2}\right)$ is a complete intersection, where $q_{1} \in S$ has order 2 , that is, $q_{1} \in \mathfrak{m}_{S}^{2} \backslash \mathfrak{m}_{S}^{3}$. Since $f$ is general, we may assume that the quadric part of $q_{1}$ has maximal rank, i.e., rank two; see also [Iarrobino 1977, Theorem 3.14]. Then, after a change of variables, $q_{1} \equiv \alpha_{1} \alpha_{2} \bmod \mathrm{m}_{S}^{3}$. Since the leading form $\alpha_{1} \alpha_{2}$ of $q_{1}$ is reducible, $q_{1}=\delta_{1} \delta_{2}$ for some $\delta_{1}, \delta_{2} \in S$ such that $\delta_{i} \equiv \alpha_{i} \bmod \mathfrak{m}_{S}^{2}$ for $i=1,2$, see, e.g., [Kunz 2005, Theorem 16.6]. After an automorphism of $S$ we may assume $\delta_{i}=\alpha_{i}$; then $\alpha_{1} \alpha_{2}=q_{1}$ annihilates $f$, so that it has the required form.

4B. Homogeneous forms and secant varieties. It is well known that if $F \in P_{s}$ is a form such that $H_{\text {Apolar }(F)}=(1,2, \ldots, 2,1)$ then the standard form of $F$ is either $x_{1}^{s}+x_{2}^{s}$ or $x_{1}^{s-1} x_{2}$. In particular, the set of such forms in $P$ is irreducible and in fact it is open in the so-called secant variety. This section is devoted to some generalizations of this result for the purposes of classifying leading forms of polynomials in $P$.

The following proposition is well known if the base field is of characteristic zero (see [Bernardi et al. 2011, Theorem 4] or [Landsberg and Ottaviani 2013]), but we could not find a reference for the positive characteristic case, so for completeness we include the proof.

Proposition 4.9. Suppose that $F \in k\left[x_{1}, x_{2}, x_{3}\right]$ is a homogeneous polynomial of degree $s \geq 4$. The following conditions are equivalent: 
(1) The algebra Apolar $(F)$ has Hilbert function $H$ beginning with $H(1)=H(2)=$ $H(3)=3$, i.e., $H=(1,3,3,3, \ldots)$.

(2) After a linear change of variables, $F$ is in one of the forms

$$
x_{1}^{s}+x_{2}^{s}+x_{3}^{s}, \quad x_{1}^{s-1} x_{2}+x_{3}^{s}, \quad \text { or } \quad x_{1}^{s-2}\left(x_{1} x_{3}+x_{2}^{2}\right) .
$$

Furthermore, the set of forms in $k\left[x_{1}, x_{2}, x_{3}\right]_{s}$ satisfying the above conditions is irreducible.

Proof. For the characteristic zero case, see [Landsberg and Ottaviani 2013] and references therein.

Let $S=k\left[\alpha_{1}, \alpha_{2}, \alpha_{3}\right]$ be a polynomial ring dual to $P$. This notation is incoherent with the global notation, but it is more readable than $S_{\text {poly }}$.

Let $I:=\operatorname{ann}_{S}(F)$ and $I_{2}:=\left\langle\theta_{1}, \theta_{2}, \theta_{3}\right\rangle \subseteq S_{2}$ be the linear space of operators of degree 2 annihilating $F$. Let $A:=S / I, J:=\left(I_{2}\right) \subseteq S$ and $B:=S / J$. Since $A$ has length greater than $3 \cdot 3>2^{3}$, the ideal $J$ is not a complete intersection. Let us analyze the Hilbert function of $A$. By symmetry of $H_{A}$, we have $H_{A}(s-1)=H_{A}(1)=3$. By Remark 2.7 we have $3=H_{A}(3) \geq H_{A}(4) \geq \cdots \geq H_{A}(s-1)=3$, thus

$$
H_{A}(m)=3 \text { for all } m=1,2, \ldots, s-1 .
$$

We will prove that the graded ideal $J$ is saturated and defines a zero-dimensional scheme of degree 3 in $\mathbb{P}^{2}=$ Proj $S$. First, $3=H_{A}(3) \leq H_{B}(3) \leq 4$ by Macaulay's growth theorem. If $H_{B}(3)=4$ then, by Lemma 2.9 and Remark 2.10, we have $H_{A}(1)=2$, a contradiction. We have proved that $H_{B}(3)=3$.

Now we want to prove that $H_{B}(4)=3$. By Macaulay's growth theorem applied to $H_{B}(3)=3$ we have $H_{B}(4) \leq 3$. If $s>4$ then $H_{A}(4)=3$, so $H_{B}(4) \geq 3$. Suppose $s=4$. By Buchsbaum-Eisenbud [1977] we know that the minimal number of generators of $I$ is odd. Moreover, we know that $A_{n}=B_{n}$ for $n<4$, thus the generators of $I$ have degree two or four. Since $I_{2}$ is not a complete intersection, there are at least two generators of degree 4 , so $H_{B}(4) \geq H_{A}(4)+2=3$.

From $H_{B}(3)=H_{B}(4)=3$, by Gotzmann's persistence theorem we see that $H_{B}(m)=3$ for all $m \geq 1$. Thus the scheme $\Gamma:=V(J) \subseteq \operatorname{Proj} k\left[\alpha_{1}, \alpha_{2}, \alpha_{3}\right]$ is finite of degree 3 and $J$ is saturated. In particular, the ideal $J=I(\Gamma)$ is contained in $I$.

We will use $\Gamma$ to compute the possible forms of $F$, in the spirit of the apolarity lemma; see [Iarrobino and Kanev 1999, Lemma 1.15]. There are four possibilities for $\Gamma$ :

(1) $\Gamma$ is a union of three distinct, noncollinear points. After a change of basis, $\Gamma=\{[1: 0: 0]\} \cup\{[0: 1: 0]\} \cup\{[0: 0: 1]\}$; then $I_{2}=\left(\alpha_{1} \alpha_{2}, \alpha_{2} \alpha_{3}, \alpha_{3} \alpha_{1}\right)$ and $F=x_{1}^{s}+x_{2}^{s}+x_{3}^{s}$.

(2) $\Gamma$ is a union of a point and scheme of length two such that $\langle\Gamma\rangle=\mathbb{P}^{2}$. After a change of basis, $I_{\Gamma}=\left(\alpha_{1}^{2}, \alpha_{1} \alpha_{2}, \alpha_{2} \alpha_{3}\right)$, so that $F=x_{3}^{s-1} x_{1}+x_{2}^{s}$. 
(3) $\Gamma$ is irreducible with support $[1: 0: 0]$ and it is not a 2 -fat point. Then $\Gamma$ is Gorenstein and so $\Gamma$ may be taken as the curvilinear scheme defined by $\left(\alpha_{3}^{2}, \alpha_{2} \alpha_{3}, \alpha_{1} \alpha_{3}-\alpha_{2}^{2}\right)$. Then, after a linear change of variables, $F=$ $x_{1}^{s-1} x_{3}+x_{2}^{2} x_{1}^{s-2}$.

(4) $\Gamma$ is a 2-fat point supported at $[1: 0: 0]$. Then $I_{\Gamma}=\left(\alpha_{2}^{2}, \alpha_{2} \alpha_{3}, \alpha_{3}^{2}\right)$, so $F=x_{1}^{s-1}\left(\lambda_{2} x_{2}+\lambda_{3} x_{3}\right)$ for some $\lambda_{2}, \lambda_{3} \in k$. But then there is a degree-one operator in $S$ annihilating $F$, a contradiction.

The set of forms $F$ which are sums of three powers of linear forms is irreducible. To see that the forms satisfying the assumptions of the proposition constitute an irreducible subset of $P_{S}$ we observe that every $\Gamma$ as above is smoothable by [Cartwright et al. 2009]. The flat family proving the smoothability of $\Gamma$ induces a family $F_{t} \rightarrow F$, such that $F_{\lambda}$ is a sum of three powers of linear forms for $\lambda \neq 0$, see [Emsalem 1978, Section C2, Corollaire]. See also [Buczyńska and Buczyński 2014] for a generalization of this method.

Proposition 4.10. Let $s \geq 4$. Consider the set $\mathcal{S}$ of all forms $F \in k\left[x_{1}, x_{2}, x_{3}, x_{4}\right]$ of degree s such that the apolar algebra of $F$ has Hilbert function $(1,4,4,4, \ldots, 4,1)$. This set is irreducible and its general member has the form $\ell_{1}^{s}+\ell_{2}^{s}+\ell_{3}^{s}+\ell_{4}^{s}$, where $\ell_{1}, \ell_{2}, \ell_{3}$ and $\ell_{4}$ are linearly independent linear forms.

Proof. First, the set $\mathcal{S}_{0}$ of forms equal to $\ell_{1}^{4}+\ell_{2}^{4}+\ell_{3}^{4}+\ell_{4}^{4}$, where $\ell_{1}, \ell_{2}, \ell_{3}$ and $\ell_{4}$ are linearly independent linear forms, is irreducible and contained in $\mathcal{S}$. Thus, it is enough to prove that $\mathcal{S}$ lies in the closure of $\mathcal{S}_{0}$.

We follow the proof of Proposition 4.9, omitting some details which can be found there. Let $S=k\left[\alpha_{1}, \alpha_{2}, \alpha_{3}, \alpha_{4}\right], I:=\operatorname{ann}_{S}(F)$ and $J:=\left(I_{2}\right)$. Set $A=S / I$ and $B=S / J$. Then $H_{B}(2)=4$ and $H_{B}(3)$ is either 4 or 5 . If $H_{B}(3)=5$ then, by Lemma 2.9, we have $H_{B}(1)=3$, a contradiction. Thus $H_{B}(3)=4$.

Now we would like to prove $H_{B}(4)=4$. By Macaulay's growth theorem, $H_{B}(4) \leq 5$. By Lemma 2.9, $H_{B}(4) \neq 5$, thus $H_{B}(4) \leq 4$. If $s>4$, then $H_{B}(4) \geq H_{A}(4) \geq 4$, so we concentrate on the case $s=4$. Let us write the minimal free resolution of $A$, which is symmetric by [Eisenbud 1995, Corollary 21.16]:

$$
\begin{aligned}
0 \rightarrow S(-8) \rightarrow S(-4)^{\oplus a} \oplus S(-6)^{\oplus 6} \rightarrow S(-3)^{\oplus b} \oplus S(-4)^{\oplus c} \oplus S(-5)^{\oplus b} & \rightarrow S(-2)^{\oplus 6} \oplus S(-4)^{\oplus a} \rightarrow S .
\end{aligned}
$$

Calculating $H_{A}(3)=4$ from the resolution, we get $b=8$. Calculating $H_{A}(4)=1$, we obtain $6-2 a+c=0$. Since $1+a=H_{B}(4) \leq 4$ we have $a \leq 3$, so $a=3, c=0$ and $H_{B}(4)=4$.

Now we calculate $H_{B}(5)$. If $s>5$ then $H_{B}(5)=4$, as before. If $s=4$ then, extracting syzygies of $I_{2}$ from the above resolution, we see that $H_{B}(5)=4+\gamma$, 
where $0 \leq \gamma \leq 8$; thus $H_{B}(5)=4$ and $\gamma=0$. If $s=5$, then the resolution of $A$ is $0 \rightarrow S(-9) \rightarrow S(-4)^{\oplus 3} \oplus S(-7)^{\oplus 6} \rightarrow S(-3)^{\oplus 8} \oplus S(-6)^{\oplus 8}$

$$
\rightarrow S(-5)^{\oplus 3} \oplus S(-2)^{\oplus 6} \rightarrow S .
$$

So $H_{B}(5)=56-20 \cdot 6+8=4$. Thus, as in the previous case, we see that $J$ is the saturated ideal of a scheme $\Gamma$ of degree 4 . Then $\Gamma$ is smoothable, by [Cartwright et al. 2009], and its smoothing induces a family $F_{t} \rightarrow F$, where $F_{\lambda} \in \mathcal{S}_{0}$ for $\lambda \neq 0$.

The following corollary is a consequence of Proposition 4.10. This corollary is not used in the proofs of the main results, but it is of certain interest of its own and shows another connection with secant varieties. For simplicity and to refer to some results from [Landsberg and Ottaviani 2013], we assume that $k=\mathbb{C}$, but the claim holds for all fields of characteristic either zero or large enough.

To state the claim we introduce catalecticant matrices. Let $\varphi_{a, s-a}: S_{a} \times P_{s} \rightarrow P_{s-a}$ be the contraction mapping applied to homogeneous polynomials of degree $s$. For $F \in P_{s}$ we obtain $\varphi_{a, s-a}(F): S_{a} \rightarrow P_{s-a}$, whose matrix is called the $a$-catalecticant matrix. It is straightforward to see that $\operatorname{rk} \varphi_{a, s-a}(F)=H_{\text {Apolar }(F)}(a)$.

Corollary 4.11. Let $s \geq 4$ and $k=\mathbb{C}$. The fourth secant variety to $s$-th Veronese reembedding of $\mathbb{P}^{n}$ is a subset $\sigma_{4}\left(v_{s}\left(\mathbb{P}^{n}\right)\right) \subseteq \mathbb{P}\left(P_{s}\right)$ set-theoretically defined by the condition $\mathrm{rk} \varphi_{a, s-a} \leq 4$, where $a=\lfloor s / 2\rfloor$.

Proof. Since $H_{\text {Apolar }(F)}(a) \leq 4$ for $F$ which is a sum of four powers of linear forms, by semicontinuity every $F \in \sigma_{4}\left(v_{s}\left(\mathbb{P}^{n}\right)\right)$ satisfies the above condition.

Let $F \in P_{s}$ be a form satisfying rk $\varphi_{a, s-a}(F) \leq 4$. Let $A=\operatorname{Apolar}(F)$ and $H=H_{A}$ be the Hilbert function of $A$. We want to reduce to the case where $H(n)=4$ for all $0<n<s$.

First we show that $H(n) \geq 4$ for all $0<n<s$. If $H(1) \leq 3$, then the claim follows from [Landsberg and Ottaviani 2013, Theorem 3.2.1(2)], so we assume $H(1) \geq 4$. Suppose that for some $n$ satisfying $4 \leq n<s$ we have $H(n)<4$. Then, by Remark 2.7, we have $H(m) \leq H(n)$ for all $m \geq n$, so that $H(1)=H(s-1)<4$, a contradiction. Thus $H(n) \geq 4$ for all $n \geq 4$. Moreover, $H(3) \geq 4$ by Macaulay's growth theorem. Suppose now that $H(2)<4$. By Theorem 2.6 the only possible case is $H(2)=3$ and $H(3)=4$. But then $H(1)=2<4$ by Lemma 2.9, a contradiction. Thus we have proved that

$$
H(n) \geq 4 \text { for all } 0<n<s .
$$

We have $H(a)=4$. If $s \geq 8$, then $a \geq 4$, so by Remark 2.7 we have $H(n) \leq 4$ for all $n>a$. Then, by the symmetry $H(n)=H(s-n)$, we have $H(n) \leq 4$ for all $n$. Together with $H(n) \geq 4$ for $0<n<s$, we have $H(n)=4$ for $0<n<s$. Then $F \in \sigma_{4}\left(v_{s}\left(\mathbb{P}^{n}\right)\right)$ by Proposition 4.10. If $a=3$ (i.e., $s=6$ or $s=7$ ), then $H(4) \leq 4$ by Lemma 2.9 and we finish the proof as in the case $s \geq 8$. If $s=5$, then $a=2$ and 
the Hilbert function of $A$ is $(1, n, 4,4, n, 1)$. Again by Lemma 2.9, we have $n \leq 4$, thus $n=4$ by (1) and Proposition 4.10 applies. If $s=4$, then $H=(1, n, 4, n, 1)$. Suppose $n \geq 5$; then Lemma 2.9 gives $n \leq 3$, a contradiction. Thus $n=4$ and Proposition 4.10 applies also to this case.

Note that, for $s \geq 8$, Corollary 4.11 was also proved, in the case $k=\mathbb{C}$, in [Buczyńska and Buczyński 2014, Theorem 1.1].

\section{Ray sums, ray families and their flatness}

Recall that $k$ is an algebraically closed field of characteristic neither 2 nor 3 . Since $k \llbracket \alpha_{i} \rrbracket$ is a discrete valuation ring, all its ideals have the form $\alpha_{i}^{v} k \llbracket \alpha_{i} \rrbracket$ for some $v \geq 0$. We use this property to construct certain decompositions of the ideals in the power series ring $S=k \llbracket \alpha_{1}, \ldots, \alpha_{n} \rrbracket$.

Definition 5.1. Let $I$ be an ideal of finite colength in the power series ring $S$ and $\pi_{i}: k \llbracket \alpha_{1}, \ldots, \alpha_{n} \rrbracket \rightarrow k \llbracket \alpha_{i} \rrbracket$ be the projection defined by $\pi_{i}\left(\alpha_{j}\right)=0$ for $j \neq i$ and $\pi_{i}\left(\alpha_{i}\right)=\alpha_{i}$.

The $i$-th ray order of $I$ is a nonnegative integer $v=\operatorname{rord}_{i}(I)$ such that $\pi_{i}(I)=\left(\alpha_{i}^{v}\right)$.

By the discussion above, the ray order is well defined. Below, by $\mathfrak{p}_{i}$ we denote the kernel of $\pi_{i}$; this is the ideal generated by all variables except for $\alpha_{i}$.

Definition 5.2. Let $I$ be an ideal of finite colength in the power series ring $S$. A ray decomposition of $I$ with respect to $\alpha_{i}$ consists of an ideal $J \subseteq S$ such that $J \subseteq I \cap \mathfrak{p}_{i}$ together with an element $q \in \mathfrak{p}_{i}$ and $v \in \mathbb{Z}_{+}$such that

$$
I=J+\left(\alpha_{i}^{\nu}-q\right) S .
$$

Note that from Definition 5.1 it follows that, for every $I$ and $i$, a ray decomposition (with $J=I \cap \mathfrak{p}_{i}$ ) exists and that $v=\operatorname{rord}_{i}(I)$ for every ray decomposition.

Definition 5.3. Let $S_{\text {poly }}=k\left[\alpha_{1}, \ldots, \alpha_{n}\right] \subseteq S$. Let $I=J+\left(\alpha_{i}^{\nu}-q\right) S$ be a ray decomposition of a finite colength ideal $I \subseteq S$. Let $J_{\text {poly }}=J \cap S_{\text {poly }}$. The associated lower ray family is

$$
k[t] \rightarrow \frac{S_{\text {poly }}[t]}{J_{\text {poly }}[t]+\left(\alpha_{i}^{v}-t \cdot \alpha_{i}-q\right) S_{\text {poly }}[t]},
$$

and the associated upper ray family is

$$
k[t] \rightarrow \frac{S_{\text {poly }}[t]}{J_{\text {poly }}[t]+\left(\alpha_{i}^{v}-t \cdot \alpha_{i}^{\nu-1}-q\right) S_{\text {poly }}[t]} .
$$

If the lower (upper) family is flat over $k[t]$ we will call it a lower (upper) ray degeneration.

Note that the lower and upper ray degenerations agree for $v=2$. 
Remark 5.4. In all considered cases the quotient $S_{\text {poly }} / J_{\text {poly }}$ will be finite over $k$, so that every ray family will be finite over $k[t]$. Then every ray degeneration will give a morphism to the Hilbert scheme. We leave this to the reader.

Remark 5.5. In this remark for simplicity we assume that $i=1$ in Definition 5.3. Below we write $\alpha$ instead of $\alpha_{1}$. Let us look at the fibers of the upper ray family from this definition in a special case, when $\alpha \cdot q \in J$. The fiber over $t=0$ is isomorphic to $S / I$. Let us take $\lambda \neq 0$ and analyze the fiber at $t=\lambda$. This fiber is supported at $(0,0, \ldots, 0)$ and at $(0, \ldots, 0, \lambda, 0, \ldots, 0)$, where $\lambda$ appears in the $i$-th position. In particular, this shows that the existence of an upper ray degeneration proves that the algebra $S / I$ is limit-reducible; this is true also for the lower ray degeneration.

Now, $\alpha^{v+1}-\lambda \alpha^{v}$ is in the ideal defining the fiber of the upper ray family over $t=\lambda$. One may compute that, near $(0, \ldots, 0)$, the ideal defining the fiber is $\left(\lambda \alpha^{\nu-1}-q\right)+J$. Similarly, near $(0, \ldots, 0, \lambda, 0, \ldots, 0)$ it is $(\alpha-\lambda)+(q)+J$. The argument is similar to (though easier than) the proof of Proposition 5.10.

Most of the families constructed in [Cartwright et al. 2009; Casnati and Notari 2009] are ray degenerations.

Definition 5.6. For a nonzero polynomial $f \in P$ and $d \geq 2$, the $d$-th ray sum of $f$ with respect to a derivation $\partial \in \mathfrak{m}_{S}$ is a polynomial $g \in P[x]$ given by

$$
\left.\left.\left.g=f+x^{d} \cdot \partial\right\lrcorner f+x^{2 d} \cdot \partial^{2}\right\lrcorner f+x^{3 d} \cdot \partial^{3}\right\lrcorner f+\cdots .
$$

The following proposition shows that a ray sum naturally induces a ray decomposition, which can be computed explicitly.

Proposition 5.7. Let $g$ be the $d$-th ray sum of $f$ with respect to $\partial \in \mathfrak{m}_{S}$ such that $\partial\lrcorner f \neq 0$. Let $\alpha$ be an element dual to $x$, so that $P[x]$ and $T:=S \llbracket \alpha \rrbracket$ are dual. The annihilator of $g$ in $T$ is given by the formula

$$
\left.\operatorname{ann}_{T}(g)=\operatorname{ann}_{S}(f)+\left(\sum_{i=1}^{d-1} k \alpha^{i}\right) \operatorname{ann}_{S}(\partial\lrcorner f\right)+\left(\alpha^{d}-\partial\right) T,
$$

where the sum is of $k$-vector spaces. In particular, the ideal $\operatorname{ann}_{T}(g) \subseteq T$ is generated by $\left.\operatorname{ann}_{S}(f), \alpha \operatorname{ann}_{S}(\partial\lrcorner f\right)$ and $\alpha^{d}-\partial$. The formula (2) is a ray decomposition of $\operatorname{ann}_{T}(g)$ with respect to $\alpha$ and with $\left.J=\operatorname{ann}_{S}(f) T+\alpha \operatorname{ann}_{S}(\partial\lrcorner f\right) T$ and $q=\partial$. Proof. It is straightforward to see that the right-hand side of (2) lies in $\operatorname{ann}_{T}(g)$. Let us take any $\partial^{\prime} \in \operatorname{ann}_{T}(g)$. Reducing the powers of $\alpha$ using $\alpha^{d}-\partial$, we can write

$$
\partial^{\prime}=\sigma_{0}+\sigma_{1} \alpha+\cdots+\sigma_{k-1} \alpha^{d-1},
$$

where the $\sigma_{\bullet}$ do not contain $\alpha$. The action of this derivation on $g$ gives

$$
\left.\left.\left.\left.0=\sigma_{0}\right\lrcorner f+x \sigma_{d-1} \partial\right\lrcorner f+x^{2} \sigma_{d-2} \partial\right\lrcorner f+\cdots+x^{d-1} \sigma_{1} \partial\right\lrcorner f+x^{d}(\cdots) .
$$


We see that $\sigma_{0} \in \operatorname{ann}_{S}(f)$ and $\left.\sigma_{i} \in \operatorname{ann}_{S}(\partial\lrcorner f\right)$ for $i \geq 1$, so the equality is proved. It is also clear that $J \subseteq \mathfrak{m}_{S} T$ and $\operatorname{ann}_{T}(g)=J+\left(\alpha^{d}-\partial\right) T$, so that indeed we obtain a ray decomposition.

Remark 5.8. It is not hard to compute the Hilbert function of the apolar algebra of a ray sum in some special cases. We mention one such case below. Let $f \in P$ be a polynomial satisfying $f_{2}=f_{1}=f_{0}=0$ and $\partial \in \mathfrak{m}_{S}^{2}$ be such that $\left.\partial\right\lrcorner f=\ell$ is a linear form, so that $\left.\partial^{2}\right\lrcorner f=0$. Let $A=\operatorname{Apolar}(f)$ and $B=\operatorname{Apolar}\left(f+x^{2} \ell\right)$. The only different values of $H_{A}$ and $H_{B}$ are $H_{B}(m)=H_{A}(m)+1$ for $m=1,2$. The assumption $f_{2}=f_{1}=f_{0}=0$ is needed to ensure that the degrees of $\left.\partial\right\lrcorner f$ and $\partial\lrcorner\left(f+x^{2} \ell\right)$ are equal for all $\partial$ not annihilating $f$.

\section{A. Flatness of ray families.}

Proposition 5.9. Let $g$ be the $d$-th ray sum with respect to $f$ and $\partial$. Then the corresponding upper and lower ray families are flat. Recall that these families are explicitly given as

$$
\begin{gathered}
k[t] \rightarrow \frac{T_{\mathrm{poly}}[t]}{J_{\mathrm{poly}}[t]+\left(\alpha^{d}-t \alpha^{d-1}-\partial\right) T_{\mathrm{poly}}[t]} \quad \text { (upper ray family), } \\
k[t] \rightarrow \frac{T_{\mathrm{poly}}[t]}{J_{\mathrm{poly}}[t]+\left(\alpha^{d}-t \alpha-\partial\right) T_{\mathrm{poly}}[t]} \quad \text { (lower ray family), }
\end{gathered}
$$

where $T_{\text {poly }}$ is the fixed polynomial subring of $T$.

Proof. We start by proving the flatness of family (4).

We want to use Proposition 2.12. To simplify notation let $J:=J_{\text {poly }}$. Denote by $\mathfrak{I}$ the ideal defining the family and suppose that some $z \in \mathfrak{I}$ lies in $(t-\lambda)$ for some $\lambda \in k$. Write $z$ as $i+i_{2}\left(\alpha^{d}-t \alpha-\partial\right)$, where $i \in J[t], i_{2} \in T_{\text {poly }}[t]$, and note that by Remark 2.13 we may assume $i \in J$ and $i_{2} \in T_{\text {poly. Since }} z \in(t-\lambda)$, we have that $i+i_{2}\left(\alpha_{1}^{v}-t \alpha_{1}^{\nu-1}-q\right)=0$, so

$$
i_{2}\left(\alpha^{d}-\lambda \alpha-\partial\right)=-i \in J .
$$

By Proposition 5.7 the ideal $J$ is homogeneous with respect to grading by $\alpha$. More precisely it is equal to $J_{0}+J_{1} \alpha$, where $J_{0}=\operatorname{ann}_{S}(f) T$ and $\left.J_{1}=\operatorname{ann}_{S}(\partial\lrcorner f\right) T$ are generated by elements not containing $\alpha$, so that $J$ is generated by elements of $\alpha$-degree at most one. We now check the assumptions of Lemma 2.15. Note that $\partial J \subseteq J_{0}$ by definition of $J$. If $r \in T_{\text {poly }}$ is such that $r \alpha^{d} \in J$, then $r \in J_{1}$, so that $r(\lambda \alpha+\partial) \in \alpha J_{1}+J_{0} \subseteq J$. Therefore, the assumptions are satisfied and the lemma shows that $i_{2} \alpha^{d} \in J$. Then $i_{2} \alpha \in J$; thus $i_{2}\left(\alpha^{d}-t \alpha\right) \in J[t] \subseteq\left(\mathfrak{I} \cap T_{\text {poly }}\right)[t]$. Since $i_{2} \partial \in \mathfrak{I} \cap T_{\text {poly }}$ by definition, this implies that $i+i_{2}\left(\alpha^{d}-t \alpha-\partial\right) \in J[t] \subseteq\left(\mathfrak{I} \cap T_{\text {poly }}\right)[t]$. Now the flatness follows from Proposition 2.12. 
The same proof works equally well for the upper ray family: one should just replace $\alpha$ by $\alpha^{d-1}$ in appropriate places of the proof. For this reason, we leave the case of family (3) to the reader.

Proposition 5.10. Let us keep the notation of Proposition 5.9. Let $\lambda \in k \backslash\{0\}$. The fibers of family (3) and family (4) over $t-\lambda$ are reducible.

Suppose that $\left.\partial^{2}\right\lrcorner f=0$ and the characteristic of $k$ does not divide $d-1$. The fiber of the family (4) over $t-\lambda$ is isomorphic to

$$
\operatorname{Spec} \operatorname{Apolar}(f) \sqcup(\operatorname{Spec} \operatorname{Apolar}(\partial f))^{\sqcup d-1} .
$$

Proof. For both families the support of the fiber over $t-\lambda$ contains the origin. The support of the fiber of family (3) contains, furthermore, a point with $\alpha=\lambda$ and other coordinates equal to zero. The support of the fiber of family (4) contains a point with $\alpha=\omega$, where $\omega^{d-1}=\lambda$.

Now let us concentrate on family (4) and on the case $\left.\partial^{2}\right\lrcorner f=0$. The support of the fiber over $t-\lambda$ is $(0, \ldots, 0,0)$ and $(0, \ldots, 0, \omega)$, where $\omega^{d-1}=\lambda$ are $(d-1)$-st roots of $\lambda$, which are pairwise different because of the characteristic assumption. We will analyze the support point by point. By hypothesis, $\left.\partial \in \operatorname{ann}_{S}(\partial\lrcorner f\right)$, so that $\alpha \cdot \partial \in J$; thus $\alpha^{d+1}-\lambda \cdot \alpha^{2}$ is in the ideal $I$ of the fiber over $t=\lambda$.

Near $(0,0, \ldots, 0)$ the element $\alpha^{d-1}-\lambda$ is invertible, so $\alpha^{2}$ is in the localization of the ideal $I$, thus $\alpha+\lambda^{-1} \partial$ is in the ideal. Now we check that the localization of $I$ is equal to $\operatorname{ann}_{S}(f)+\left(\alpha+\lambda^{-1} \partial\right) T_{\text {poly }}$. Explicitly, one should check that

$$
\left(\operatorname{ann}_{S}(f)+\left(\alpha+\lambda^{-1} \partial\right) T_{\text {poly }}\right)_{(0, \ldots, 0)}=\left(\operatorname{ann}_{S}(f)+\left(\alpha^{d}-\lambda \alpha-\partial\right) T_{\text {poly }}\right)_{(0, \ldots, 0)} .
$$

Then the stalk of the fiber at $(0, \ldots, 0)$ is isomorphic to Spec Apolar $(f)$.

Near $(0,0, \ldots, 0, \omega)$, the elements $\alpha$ and $\left(\alpha^{k+1}-\lambda \cdot \alpha^{2}\right) /(\alpha-\omega)$ are invertible, so $\left.\operatorname{ann}_{S}(\partial\lrcorner f\right)$ and $\alpha-\omega$ are in the localization of $I$. This, along with the other inclusion, proves that this localization is generated by $\left.\operatorname{ann}_{S}(\partial\lrcorner f\right)$ and $\alpha-\omega$ and thus the stalk of the fiber is isomorphic to $\operatorname{Spec} \operatorname{Apolar}(\partial f)$.

We make the most important corollary explicit:

Corollary 5.11. We keep the notation of Proposition 5.9. Suppose that char $k$ does not divide $d-1$ and $\left.\partial^{2}\right\lrcorner f=0$. If both apolar algebras of $f$ and $\left.\partial\right\lrcorner f$ are smoothable then also the apolar algebra of every ray sum of $f$ with respect to $\partial$ is smoothable.

Example 5.12. Let $f \in k\left[x_{1}, \ldots, x_{n}\right]$ be a dual socle generator of an algebra $A$. Then the algebra $B=\operatorname{Apolar}\left(f+x_{n+1}^{2}\right)$ is limit-reducible: it is a limit of algebras of the form $A \times k$. In particular, if $A$ is smoothable, then $B$ is also smoothable.

Combining this with Proposition 4.5, we see that every local Gorenstein algebra $A$ of socle degree $s$ with $\Delta_{A, s-2}=(0, q, 0)$, where $q \neq 0$, is limit-reducible. 
If $\operatorname{deg} f \geq 2$ then the Hilbert functions of the algebras $A=\operatorname{Apolar}(f)$ and $B=$ $\operatorname{Apolar}\left(f+x_{n+1}^{2}\right)$ are related by $H_{B}(m)=H_{A}(m)$ for $m \neq 1$ and $H_{B}(1)=H_{A}(1)+1$.

Above, we took advantage of the explicit form of ray decompositions coming from ray sums to analyze the resulting ray families in depth. In Proposition 5.13 below we prove the flatness of the upper ray family without such knowledge. The price paid for this is the fact that we get no information about the fibers of this family.

Proposition 5.13. Let $f=x_{1}^{s}+g \in P$ be a polynomial of degree s such that $\left.\alpha_{1}^{c}\right\lrcorner g=0$ for some $c$ satisfying $2 c \leq s$. Then any ray decomposition $\operatorname{ann}_{S}(f)=\left(\alpha_{1}^{v}-q\right)+J$, where $J=\operatorname{ann}_{S}(f) \cap\left(\alpha_{2}, \ldots, \alpha_{n}\right)$, gives rise to an upper ray degeneration. In particular, Apolar $(f)$ is limit-reducible.

Proof. Let $\mathfrak{I}:=\left(\alpha_{1}^{\nu}-t \alpha_{1}^{\nu-1}-q\right)+J$ be the ideal defining the ray family and recall that $q, J \subseteq \mathfrak{p}_{1}$, where $\mathfrak{p}_{1}=\left(\alpha_{2}, \ldots, \alpha_{n}\right)$.

Since $\alpha_{1}^{v}-q \in \operatorname{ann}_{S}(f)$, we have $\left.\left.\left.\left.q\right\lrcorner g=q\right\lrcorner f=\alpha_{1}^{v}\right\lrcorner f=x_{1}^{s-v}+\alpha_{1}^{v}\right\lrcorner g$. Then $\left.\left.\left.\alpha_{1}^{s-v}(q\lrcorner g\right)=\alpha_{1}^{s-v}\right\lrcorner x_{1}^{s-v}+\alpha_{1}^{s}\right\lrcorner g=1$; thus $\left.\alpha_{1}^{s-v}\right\lrcorner g \neq 0$. It follows that $s-v \leq c-1$, so $v-1 \geq s-c \geq c$; thus $\left.\alpha_{1}^{\nu-1}\right\lrcorner g=0$. For all $\gamma \in \mathfrak{p}_{1}$, we claim that

$$
\gamma \cdot\left(\alpha_{1}^{\nu}-t \alpha_{1}^{\nu-1}-q\right) \in J[t] .
$$

Note that $\left.\left(\alpha_{1}^{\nu}-q\right)\right\lrcorner f=0$ and $\left.\left.\alpha_{1}^{\nu-1} \gamma\right\lrcorner f=\alpha_{1}^{\nu-1} \gamma\right\lrcorner g=0$. This means that $\alpha_{1}^{\nu-1} \gamma \in J$. Since $\left(\alpha_{1}^{\nu}-q\right) \gamma \in J$ always, we have proved (5).

Let $\mathfrak{I} \subseteq S_{\text {poly }}[t]$ be the ideal defining the upper ray family. Take any $\lambda \in k$ and an element $i \in \mathfrak{I} \cap(t-\lambda)$. We will prove that $i \in \mathfrak{I}(t-\lambda)+\mathfrak{I}_{0}[t]$, where $\mathfrak{I}_{0}=\mathfrak{I} \cap S$, then Proposition 2.12 asserts that $S[t] / \mathfrak{I}$ is flat. Write $i=i_{1}+i_{2}\left(\alpha_{1}^{\nu}-t \alpha_{1}^{\nu-1}-q\right)$. As before, we may assume $i_{1} \in J$ and $i_{2} \in S$. Since $i \in(t-\lambda)$, we have $i_{1}+i_{2}\left(\alpha_{1}^{\nu}-\lambda \alpha_{1}^{\nu-1}-q\right)=0$. Since $i_{1} \in \mathfrak{p}_{1}$, we also have $i_{2} \in \mathfrak{p}_{1}$. But then by inclusion (5) we have $i_{2}\left(\alpha_{1}^{\nu}-t \alpha_{1}^{\nu-1}-q\right) \subseteq \mathfrak{I}_{0}[t]$. Since clearly $i_{1} \in J \subseteq \mathfrak{I}_{0}[t]$, the assumptions of Proposition 2.12 are satisfied; thus the upper ray family is flat.

Now, Remark 5.5 shows that a general fiber of the upper ray degeneration is reducible; thus, Apolar $(f)$ is a flat limit of reducible algebras, so limit-reducible.

Example 5.14. Let $f \in k\left[x_{1}, x_{2}, x_{3}, x_{4}\right]$ be a polynomial of degree 4. Suppose that the leading form $f_{4}$ of $f$ can be written as $f_{4}=x_{1}^{4}+g_{4}$, where $g_{4} \in k\left[x_{2}, x_{3}, x_{4}\right]$. We will prove that Apolar $(f)$ is limit-reducible. By Example 4.3 we may assume that $f=x_{1}^{4}+g$, where $\left.\alpha_{1}^{2}\right\lrcorner g=0$. By Proposition 5.13 we see that $\operatorname{Apolar}(f)$ is limit-reducible.

Example 5.15. Suppose that an Artin local Gorenstein algebra $A$ has Hilbert function $H_{A}=\left(1, H_{1}, \ldots, H_{c}, 1, \ldots, 1\right)$ and socle degree $s \geq 2 c$. By Example 4.4 we may assume that $A \simeq \operatorname{Apolar}\left(x_{1}^{s}+g\right)$, where $\left.\alpha_{1}^{c}\right\lrcorner g=0$ and $\operatorname{deg} g \leq c+1$. Then 
by Proposition 5.13 we obtain a flat degeneration

$$
k[t] \rightarrow \frac{S[t]}{\left(\alpha_{1}^{v}-t \alpha_{1}^{\nu-1}-q\right)+J} .
$$

Thus $A$ is limit-reducible in the sense of Definition 2.5. Let us take $\lambda \neq 0$. By Remark 5.5 the fiber over $t=\lambda$ is supported at $(0,0, \ldots, 0)$ and at $(\lambda, 0, \ldots, 0)$ and the ideal defining this fiber near $(0,0, \ldots, 0)$ is $I_{0}=\left(\lambda \alpha_{1}^{\nu-1}-q\right)+J$. From the proof of 5.13 it follows that $\left.\alpha_{1}^{\nu-1}\right\lrcorner g=0$. Then one can check that $I_{0}$ lies in the annihilator of $\lambda^{-1} x_{1}^{s-1}+g$. Since $\left.\left.\sigma\right\lrcorner\left(x_{1}^{s}+g\right)=\sigma\right\lrcorner\left(\lambda^{-1} x_{1}^{s-1}+g\right)$ for every $\sigma$ in $\left(\alpha_{2}, \ldots, \alpha_{n}\right)$, one calculates that the apolar algebra of $\lambda^{-1} x_{1}^{s-1}+g$ has Hilbert function $\left(1, H_{1}, \ldots, H_{c}, 1, \ldots, 1\right)$ and socle degree $s-1$. Then $\operatorname{dim}_{k} \operatorname{Apolar}\left(x_{1}^{s-1}+g\right)=$ $\operatorname{dim}_{k} \operatorname{Apolar}\left(\lambda^{-1} x_{1}^{s}+g\right)-1$. Therefore, the fiber is a union of a point and of Spec Apolar $\left(\lambda^{-1} x_{1}^{s}+g\right)$, i.e., the degeneration (6) peels one point off $A$.

5B. Tangent-preserving ray degenerations. A (finite) ray degeneration gives a morphism from Spec $k[t]$ to the Hilbert scheme, that is, a curve on the Hilbert scheme $\mathcal{H i l b}\left(\mathbb{P}^{n}\right)$. In this section we prove that in some cases the dimension of the tangent space to $\mathcal{H} i l b\left(\mathbb{P}^{n}\right)$ is constant along this curve. This enables us to prove that certain points of this scheme are smooth without the need for lengthy computations.

This section seems to be the most technical part of the paper, so we include even more examples. The most important results here are Theorem 5.18 together with Corollary 5.20; see examples below Corollary 5.20 for applications.

Recall (e.g., [Jelisiejew 2014a, Proposition 4.10] or [Casnati and Notari 2009]) that the dimension of the tangent space to $\mathcal{H i l b}\left(\mathbb{P}^{n}\right)$ at a $k$-point corresponding to a Gorenstein scheme Spec $S / I$ is $\operatorname{dim}_{k} S / I^{2}-\operatorname{dim}_{k} S / I$.

Lemma 5.16. Let $d \geq 2$. Let $g$ be the $d$-th ray sum of $f \in P$ with respect to $\partial \in S$ such that $\left.\partial^{2}\right\lrcorner f=0$. Denote $I:=\operatorname{ann}_{S}(f)$ and $\left.J:=\operatorname{ann}_{S}(\partial\lrcorner f\right)$. Take $T=S \llbracket \alpha \rrbracket$ to be the ring dual to $P[x]$ and let

$$
\mathfrak{I}:=\left(I+J \alpha+\left(\alpha^{d}-t \alpha-\partial\right)\right) \cdot T[t]
$$

be the ideal in $T[t]$ defining the associated lower ray degeneration; see Proposition 5.9. Then the family $k[t] \rightarrow T[t] / \mathfrak{I}^{2}$ is flat if and only if $\left(I^{2}: \partial\right) \cap I \cap J^{2} \subseteq I \cdot J$.

Proof. To prove flatness we will use Proposition 2.12. Take an element $i \in \mathfrak{I}^{2} \cap(t-\lambda)$. We want to prove that $i \in \mathfrak{I}^{2}(t-\lambda)+\mathfrak{I}_{0}[t]$, where $\mathfrak{I}_{0}[t]=\mathfrak{I}^{2} \cap T$. Let $\mathcal{J}:=(I+J \alpha) T$. Subtracting a suitable element of $\mathfrak{I}^{2}(t-\lambda)$, we may assume that

$$
i=i_{1}+i_{2}\left(\alpha^{d}-t \alpha-\partial\right)+i_{3}\left(\alpha^{d}-t \alpha-\partial\right)^{2},
$$

where $i_{1} \in \mathcal{J}^{2}, i_{2} \in \mathcal{J}$ and $i_{3} \in T$. We will in fact show that $i \in \mathfrak{I}^{2}(t-\lambda)+\mathcal{J}^{2}[t]$. 
To simplify notation, denote $\sigma=\alpha^{d}-\lambda \alpha-\partial$. Note that $J \sigma \subseteq \mathcal{J}$. We have $i_{1}+i_{2} \sigma+i_{3} \sigma^{2}=0$. Let $j_{3}:=i_{3} \sigma$. We want to apply Lemma 2.15 ; below, we check its assumptions. The ideal $\mathcal{J}$ is homogeneous with respect to $\alpha$, generated in degrees less than $d$. Let $s \in T$ be an element satisfying $s \alpha^{d} \in \mathcal{J}$. Then $s \in J$, which implies $s(\lambda \alpha+\partial) \in \mathcal{J}$. By Lemma 2.15 and $i_{3} \sigma^{2}=j_{3} \sigma \in \mathcal{J}$ we obtain $j_{3} \alpha^{d} \in \mathcal{J}$, so $i_{3} \sigma \alpha^{d} \in \mathcal{J}$. Applying the same argument to $i_{3} \alpha^{d}$ we obtain $i_{3} \alpha^{2 d} \in \mathcal{J}$, therefore $i_{3} \in J T$. Then

$$
\begin{aligned}
i_{3}\left(\alpha^{d}-t \alpha-\partial\right)^{2}-i_{3} \sigma\left(\alpha^{d}-t \alpha-\partial\right) & =i_{3} \alpha(t-\lambda)\left(\alpha^{d}-t \alpha-\partial\right) \\
& \in \mathcal{J}(t-\lambda)\left(\alpha^{d}-t \alpha-\partial\right) \subseteq \mathfrak{I}^{2}(t-\lambda) .
\end{aligned}
$$

Subtracting this element from $i$ and substituting $i_{2}:=i_{2}+i_{3} \sigma$, we may assume $i_{3}=0$. We obtain

$$
0=i_{1}+i_{2} \sigma=i_{1}+i_{2}\left(\alpha^{d}-\lambda \alpha-\partial\right) .
$$

Let $i_{2}=j_{2}+v_{2} \alpha$, where $j_{2} \in S$, i.e., it does not contain $\alpha$. Since $i_{2} \in \mathcal{J}$, we have $j_{2} \in I$. As before, we have $v_{2} \alpha\left(\left(\alpha^{d}-t \alpha-\partial\right)-\sigma\right)=v_{2} \alpha^{2}(t-\lambda) \in \mathfrak{I}^{2}(t-\lambda)$, so that we may assume $v_{2}=0$.

Comparing the top $\alpha$-degree terms of (7), we see that $j_{2} \in J^{2}$. Comparing the terms of (7) not containing $\alpha$, we deduce that $j_{2} \partial \in I^{2}$; thus $j_{2} \in\left(I^{2}: \partial\right)$. Jointly, $j_{2} \in I \cap J^{2} \cap\left(I^{2}: \partial\right)$; thus $j_{2} \in I J$ by assumption. But then $j_{2} \alpha \in \mathcal{J}^{2}$, so $j_{2}\left(\alpha^{d}-t \alpha-\partial\right) \in \mathcal{J}^{2}[t]$ and, since $i_{1} \in \mathcal{J}^{2}$, the element $i$ lies in $\mathcal{J}^{2}[t] \subseteq \Im_{0}[t]$. Thus the assumptions of Proposition 2.12 are satisfied and the family $T[t] / \mathfrak{I}^{2}$ is flat over $k[t]$.

The converse is easier: one takes $i_{2} \in I \cap J^{2} \cap\left(I^{2}: \partial\right)$ such that $i_{2} \notin I J$. On one hand, the element $j:=i_{2}\left(\alpha^{d}-\partial\right)$ lies in $\mathcal{J}^{2}$ and we get that $i_{2}\left(\alpha^{d}-t \alpha-\partial\right)-j=$ $t i_{2} \alpha \in \mathfrak{I}^{2}$. On the other hand, if $i_{2} \alpha \in \mathfrak{I}^{2}$, then $i_{2} \alpha \in\left(\mathfrak{I}^{2}+(t)\right) \cap T=\left(\mathcal{J}+\left(\alpha^{d}-\partial\right)\right)^{2}$, which is not the case.

Remark 5.17. Let us keep the notation of Lemma 5.16. Fix $\lambda \in k \backslash\{0\}$ and suppose that the characteristic of $k$ does not divide $d-1$. The supports of the fibers of $S[t] / \mathfrak{I}, \quad \mathfrak{I} / \mathfrak{I}^{2}$ and $S[t] / \mathfrak{I}^{2}$ over $t=\lambda$ are finite and equal. In particular, from Proposition 5.10 it follows that the dimension of the fiber of $\mathfrak{I} / \mathfrak{I}^{2}$ over $t-\lambda$ is equal to $\tan (f)+(d-1) \tan (\partial\lrcorner f)$, where $\tan (h)=\operatorname{dim}_{k} \operatorname{ann}_{S}(h) / \operatorname{ann}_{S}(h)^{2}$ is the dimension of the tangent space to the point of the Hilbert scheme corresponding to Spec $S / \operatorname{ann}_{S}(h)$.

Theorem 5.18. Suppose that a polynomial $f \in P$ corresponds to a smoothable, unobstructed algebra Apolar $(f)$. Let $\partial \in S$ be such that $\left.\partial^{2}\right\lrcorner f=0$ and the algebra $\operatorname{Apolar}(\partial\lrcorner f)$ is smoothable and unobstructed. The following are equivalent:

(1) The d-th ray sum of $f$ with respect to $\partial$ is unobstructed for some $d$ such that $2 \leq d \leq \operatorname{char} k($ or $2 \leq d$ if $\operatorname{char} k=0)$. 
(1a) The $d$-th ray sum of $f$ with respect to $\partial$ is unobstructed for all $d$ such that $2 \leq d \leq \operatorname{char} k($ or $2 \leq d$ if $\operatorname{char} k=0)$.

(2) The $k[t]$-module $\mathfrak{I} / \mathfrak{I}^{2}$ is flat, where $\mathfrak{I}$ is the ideal defining the lower ray family of the $d$-th ray sum for some $2 \leq d \leq$ char $k$ (or $2 \leq d$ if char $k=0$ ); see Definition 5.3.

(2a) The $k[t]$-module $\mathfrak{I} / \mathfrak{I}^{2}$ is flat, where $\mathfrak{I}$ is the ideal defining the lower ray family of the $d$-th ray sum for every $2 \leq d \leq$ char $k$ (or $2 \leq d$ if char $k=0$ ); see Definition 5.3.

(3) The family $k[t] \rightarrow S[t] / \mathfrak{I}^{2}$ is flat, where $\mathfrak{I}$ is the ideal defining the lower ray family of the $d$-th ray sum for some $2 \leq d \leq$ char $k$ (or $2 \leq d$ if char $k=0$ ).

(3a) The family $k[t] \rightarrow S[t] / \mathfrak{I}^{2}$ is flat, where $\mathfrak{I}$ is the ideal defining the lower ray family of the $d$-th ray sum for every $2 \leq d \leq \operatorname{char} k$ (or $2 \leq d$ if $\operatorname{char} k=0$ ).

(4) The following inclusion (equivalent to equality) of ideals in $S$ holds: $I \cap J^{2} \cap$ $\left(I^{2}: \partial\right) \subseteq I \cdot J$, where $I=\operatorname{ann}_{S}(f)$ and $\left.J=\operatorname{ann}_{S}(\partial\lrcorner f\right)$.

Proof. It is straightforward to check that the inclusion $I \cdot J \subseteq I \cap J^{2} \cap\left(I^{2}: \partial\right) \subseteq I \cdot J$ in point (4) always holds, thus the other inclusion is equivalent to equality.

(3) $\Longleftrightarrow(4) \Longleftrightarrow$ (3a): The equivalence of (3) and (4) follows from Lemma 5.16. Since (4) is independent of $d$, the equivalence of (4) and (3a) also follows.

$(2) \Longleftrightarrow(3)$ and $(2 \mathrm{a}) \Longleftrightarrow(3 \mathrm{a})$ : We have an exact sequence of $k[t]$-modules

$$
0 \rightarrow \mathfrak{I} / \mathfrak{I}^{2} \rightarrow S[t] / \mathfrak{I}^{2} \rightarrow S[t] / \mathfrak{I} \rightarrow 0 .
$$

Since $S[t] / \mathfrak{I}$ is a flat $k[t]$-module by Proposition 5.9, we see from the long exact sequence of Tor that $\mathfrak{I} / \mathfrak{I}^{2}$ is flat if and only if $S[t] / \mathfrak{I}^{2}$ is flat.

$(1) \Longleftrightarrow(2)$ and $(1 \mathrm{a}) \Longleftrightarrow(2 \mathrm{a})$ : Let $g \in P[x]$ be the $d$-th ray sum of $f$ with respect to $\partial$. We may consider Apolar $(g), \operatorname{Apolar}(f)$ and $\operatorname{Apolar}(\partial\lrcorner f)$ as quotients of a polynomial ring $T_{\text {poly }}$, corresponding to points of the Hilbert scheme. The dimension of the tangent space at $\operatorname{Apolar}(g)$ is given by $\operatorname{dim}_{k} \mathfrak{I} / \mathfrak{I}^{2} \otimes k[t] / t=\operatorname{dim}_{k} \mathfrak{I} /\left(\mathfrak{I}^{2}+(t)\right)$. By Remark 5.17 it is equal to the sum of the dimension of the tangent space at Apolar $(f)$ and $d-1$ times the dimension of the tangent space to $\operatorname{Apolar}(\partial\lrcorner f)$. Since both algebras are smoothable and unobstructed, we conclude that Apolar $(g)$ is also unobstructed. On the other hand, if Apolar $(g)$ is unobstructed, then $\mathfrak{I} / \mathfrak{I}^{2}$ is a finite $k[t]$-module such that the length of the fiber $\mathfrak{I} / \mathfrak{I}^{2} \otimes k[t] / \mathfrak{m}$ does not depend on the choice of the maximal ideal $\mathfrak{m} \subseteq k[t]$. Then $\mathfrak{I} / \mathfrak{I}^{2}$ is flat, by [Hartshorne 1977, Exercise II.5.8 or Theorem III.9.9] applied to the associated sheaf.

Remark 5.19. The condition from point (4) of Theorem 5.18 seems very technical. It is enlightening to look at the images of $\left(I^{2}: \partial\right) \cap I$ and $I \cdot J$ in $I / I^{2}$. The image of $\left(I^{2}: \partial\right) \cap I$ is the annihilator of $\partial$ in $I / I^{2}$. This annihilator clearly contains 
$(I: \partial) \cdot I / I^{2}=J \cdot I / I^{2}$. This shows that if the $S / I$-module $I / I^{2}$ is "nice", for example free, we should have an equality $\left(I^{2}: \partial\right) \cap I=I \cdot J$. More generally, this equality is connected to the syzygies of $I / I^{2}$.

In the remainder of this subsection we will prove that in several situations the conditions of Theorem 5.18 are satisfied.

Corollary 5.20. We keep the notation and assumptions of Theorem 5.18. Suppose further that the algebra $S / I=\operatorname{Apolar}(f)$ is a complete intersection. Then the equivalent conditions of Theorem 5.18 are satisfied.

Proof. Since $S / I$ is a complete intersection, the $S / I$-module $I / I^{2}$ is free; see, e.g., [Matsumura 1986, Theorem 16.2] and discussion above it or [Eisenbud 1995, Exercise 17.12a]. This implies that $\left(I^{2}: \partial\right) \cap I=(I: \partial) I=J I$, because $J=$ $\left.\left.\operatorname{ann}_{S}(\partial\lrcorner f\right)=\{s \in S \mid s \partial\lrcorner f=0\right\}=\left(\operatorname{ann}_{S}(f): \partial\right)=(I: \partial)$. Thus the condition from point (4) of Theorem 5.18 is satisfied.

Example 5.21. If $A=S / I$ is a complete intersection, then it is smoothable and unobstructed (see Section 2D). The apolar algebras of monomials are complete intersections, therefore the assumptions of Theorem 5.18 are satisfied, for example, for $f=x_{1}^{2} x_{2}^{2} x_{3}$ and $\partial=\alpha_{2}^{2}$. Now Corollary 5.20 implies that the equivalent conditions of the theorem are also satisfied; thus $x_{1}^{2} x_{2}^{2} x_{3}+x_{4}^{d} x_{1}^{2} x_{3}=\left(x_{2}^{2} x_{3}\right)\left(x_{1}^{2}+x_{4}^{d}\right)$ is unobstructed for every $d \geq 2$ (provided char $k=0$ or $d \leq$ char $k$ ). Similarly, $x_{1}^{2} x_{2} x_{3}+x_{4}^{2} x_{1}$ is unobstructed and has Hilbert function $(1,4,5,3,1)$.

Example 5.22. Let $f=\left(x_{1}^{2}+x_{2}^{2}\right) x_{3}$; then $\operatorname{ann}_{S}(f)=\left(\alpha_{1}^{2}-\alpha_{2}^{2}, \alpha_{1} \alpha_{2}, \alpha_{3}^{2}\right)$ is a complete intersection. Take $\partial=\alpha_{1} \alpha_{3}$, then $\left.\partial\right\lrcorner f=x_{1}$ and $\left.\partial^{2}\right\lrcorner f=0$; thus $\left.f+x_{4}^{2} \partial\right\lrcorner f=x_{1}^{2} x_{3}+x_{2}^{2} x_{3}+x_{4}^{2} x_{1}$ is unobstructed. Note that by Remark 5.8 the apolar algebra of this polynomial has Hilbert function $(1,4,4,1)$.

Proposition 5.23. Let $f \in P$ be such that Apolar $(f)$ is a complete intersection.

Let $d$ be a natural number. Suppose that char $k=0$ or $d \leq$ char $k$. Take $\partial \in S$ such that $\left.\partial^{2}\right\lrcorner f=0$ and Apolar $\left.(\partial\lrcorner f\right)$ is also a complete intersection. Let $g \in P[y]$ be the $d$-th ray sum $f$ with respect to $\partial$, i.e., $\left.g=f+y^{d} \partial\right\lrcorner f$.

Suppose that $\operatorname{deg} \partial\lrcorner f>0$. Let $\beta$ be the variable dual to $y$ and $\sigma \in S$ be such that $\sigma\lrcorner(\partial\lrcorner f)=1$. Take $\varphi:=\sigma \beta \in T=S \llbracket \beta \rrbracket$. Let $h$ be any ray sum of $g$ with respect to $\varphi$; explicitly,

$$
\left.h=f+y^{d} \partial\right\lrcorner f+z^{m} y^{d-1}
$$

for some $m \geq 2$.

Then the algebra Apolar( $h)$ is unobstructed.

Proof. First note that $\varphi\lrcorner g=y^{d-1}$ and so $\left.\left.\varphi^{2}\right\lrcorner g=\sigma\right\lrcorner y^{d-2}=0$, since $\sigma \in \mathfrak{m}_{S}$. Therefore, indeed, $h$ has the presented form. 
From Corollary 5.20 it follows that Apolar $(g)$ is unobstructed. Since $\varphi\lrcorner g=y^{d-1}$, the algebra Apolar $(\varphi\lrcorner g)$ is unobstructed as well. Now, by Theorem 5.18 it remains to prove that

$$
\left(I_{g}^{2}: \varphi\right) \cap I_{g} \cap J_{g}^{2} \subseteq I_{g} J_{g},
$$

where $\left.I_{g}=\operatorname{ann}_{T}(g), J_{g}=\operatorname{ann}_{T}(\varphi\lrcorner g\right)$. The rest of the proof is a technical verification of this claim. Denote $I_{f}:=\operatorname{ann}_{S}(f)$ and $\left.J_{f}:=\operatorname{ann}_{S}(\partial\lrcorner f\right)$; note that we take annihilators in $S$. By Proposition 5.7 we have $I_{g}=I_{f} T+\beta J_{f} T+\left(\beta^{d}-\partial\right) T$. Consider $\gamma \in T$ lying in $\left(I_{g}^{2}: \varphi\right) \cap I_{g} \cap J_{g}^{2}$. Write $\gamma=\gamma_{0}+\gamma_{1} \beta+\gamma_{2} \beta^{2}+\cdots$, where $\gamma_{i} \in S$, so they do not contain $\beta$. We will prove that $\gamma \in I_{g} J_{g}$.

First, since $\left(\beta^{d}-\partial\right)^{2} \in I_{g} J_{g}$ we may reduce powers of $\beta$ in $\gamma$ using this element and so we assume $\gamma_{i}=0$ for $i \geq 2 d$. Let us take $i<2 d$. Since $\gamma \in J_{g}^{2}=$ $\left(\operatorname{ann}_{T}\left(y^{d-1}\right)\right)^{2}=\left(\mathfrak{m}_{S}, \beta^{d}\right)^{2}$, we see that $\gamma_{i} \in \mathfrak{m}_{S} \subseteq J_{g}$. For $i>d$ we have $\beta^{i} \in I_{g}$, so that $\gamma_{i} \beta^{i} \in J_{g} I_{g}$ and we may assume $\gamma_{i}=0$. Moreover, $\beta^{d} \gamma_{d}-\partial \gamma_{d} \in I_{g} J_{g}$, so we may also assume $\gamma_{d}=0$, obtaining

$$
\gamma=\gamma_{0}+\cdots+\gamma_{d-1} \beta^{d-1} .
$$

From the explicit description of $I_{g}$ in Proposition 5.7 it follows that $\gamma_{i} \in J_{f}$ for all $i$.

Let $M=I_{g}^{2} \cap \varphi T=I_{g}^{2} \cap J_{f} \beta T$. Then, for $\gamma$ as above we have $\gamma \varphi \in M$, so we will analyze the module $M$. Recall that

$$
I_{g}^{2}=I_{f}^{2} \cdot T+\beta I_{f} J_{f} \cdot T+\beta^{2} J_{f}^{2} \cdot T+\left(\beta^{d}-\partial\right) I_{f} \cdot T+\left(\beta^{d}-\partial\right) \beta J_{f} \cdot T+\left(\beta^{d}-\partial\right)^{2} \cdot T .
$$

We claim that

$$
M \subseteq I_{f}^{2} \cdot T+\beta I_{f} J_{f} \cdot T+\beta^{2} J_{f}^{2} \cdot T+\left(\beta^{d}-\partial\right) \beta J_{f} \cdot T .
$$

We have $I_{g}^{2} \subseteq J_{f} \cdot T+\left(\beta^{d}-\partial\right)^{2} \cdot T$ so, if an element of $I_{g}^{2}$ lies in $J_{f} \cdot T$, then its coefficient associated to $\left(\beta^{d}-\partial\right)^{2}$ in presentation (9) is an element of $J_{f}$, by Lemma 2.15. Since $J_{f} \cdot\left(\beta^{d}-\partial\right) \subseteq I_{f}+\beta J_{f}$, we may ignore the term $\left(\beta^{d}-\partial\right)^{2}$ :

$$
M \subseteq I_{f}^{2} \cdot T+\beta I_{f} J_{f} \cdot T+\beta^{2} J_{f}^{2} \cdot T+\left(\beta^{d}-\partial\right) I_{f} \cdot T+\left(\beta^{d}-\partial\right) \beta J_{f} \cdot T .
$$

Choose an element of $M$ and let $i \in I_{f} \cdot T$ be the coefficient of this element associated to $\left(\beta^{d}-\partial\right)$. Since $I_{f} T \cap \beta T \subseteq J_{f} T$, we may assume that $i$ does not contain $\beta$, i.e., $i \in I_{f}$. Now, if an element of the right-hand side of (11) lies in $\beta \cdot T$, then the coefficient $i$ satisfies $i \cdot \partial \in I_{f}^{2}$, so that $i \in\left(I_{f}^{2}: \partial\right)$. Since $I_{f}$ is a complete intersection ideal, the $S / I_{f}$-module $I_{f} / I_{f}^{2}$ is free; see Corollary 5.20 for references. Then we have $\left(I_{f}^{2}: \partial\right)=\left(I_{f}: \partial\right) I_{f}$ and $i \in\left(I_{f}: \partial\right) I_{f}=I_{f} J_{f}$. Then $i \cdot\left(\beta^{d}-\partial\right) \subseteq I_{f}^{2}+\beta \cdot I_{f} \cdot J_{f}$ and so the inclusion (10) is proved. We return to the proof of proposition.

From Lemma 2.15 applied to the ideal $J_{f}^{2} T$ and the element $\beta\left(\beta^{d}-\partial\right)$, and the fact that $\beta \partial J_{f}^{2} \subseteq I_{g}^{2}$, we compute that $M \cap\left\{\delta \mid \operatorname{deg}_{\beta} \delta \leq d\right\}$ is a subset 
of $I_{f}^{2} \cdot T+\beta \cdot I_{f} J_{f} \cdot T+\beta^{2} J_{f}^{2} \cdot T$. Then $\gamma \varphi=\gamma \beta \sigma$ lies in this set, so that $\gamma_{0} \in\left(I_{f} J_{f}: \sigma\right)$ and $\gamma_{n} \in\left(J_{f}^{2}: \sigma\right)$ for $n>1$. Since Apolar $(f)$ and $\left.\operatorname{Apolar}(\partial\lrcorner f\right)$ are complete intersections, we have $\gamma_{0} \in I_{f} \mathfrak{m}_{S}$ and $\gamma_{i} \in J_{f} \mathfrak{m}_{S}$ for $i \geq 1$. It follows that $\gamma \in I_{g} \mathfrak{m}_{S} \subseteq I_{g} J_{g}$.

Example 5.24. Let $f \in P$ be a polynomial such that $A=\operatorname{Apolar}(f)$ is a complete intersection. Take $\partial$ such that $\partial\lrcorner f=x_{1}$ and $\left.\partial^{2}\right\lrcorner f=0$. Then the apolar algebra of $f+y_{1}^{d} x_{1}+y_{2}^{m} y_{1}^{d-1}$ is unobstructed for any $d, m \geq 2$ (less or equal to char $k$ if it is nonzero). In particular, $g=f+y_{1}^{2} x_{1}+y_{2}^{2} y_{1}$ is unobstructed.

Continuing Example 5.22, if $f=x_{1}^{2} x_{3}+x_{2}^{2} x_{3}$; then $x_{1}^{2} x_{3}+x_{2}^{2} x_{3}+x_{4}^{2} x_{1}+x_{5}^{2} x_{4}$ is unobstructed. The apolar algebra of this polynomial has Hilbert function $(1,5,5,1)$.

Let $g=x_{1}^{2} x_{3}+x_{2}^{2} x_{3}+x_{4}^{2} x_{1}$, then $x_{1}^{2} x_{3}+x_{2}^{2} x_{3}+x_{4}^{2} x_{1}+x_{5}^{2} x_{4}$ is a ray sum of $g$ with respect to $\partial=\alpha_{4} \alpha_{1}$. Let $I:=\operatorname{ann}_{S}(g)$ and $J:=(I: \partial)$. In contrast with Corollary 5.20 and Example 5.22 one may check that all three terms, $I, J^{2}$ and $\left(I^{2}: \partial\right)$, are necessary to obtain equality in the inclusion (8) for $g$ and $\partial$, that is, no two ideals of $I, J^{2},\left(I^{2}: \partial\right)$ have intersection equal to $I J$.

Example 5.25. Let $f=x_{1}^{5}+x_{2}^{4}$. Then the annihilator of $f$ in $k\left[\alpha_{1}, \alpha_{2}\right]$ is a complete intersection, and this is true for every $f \in k\left[x_{1}, x_{2}\right]$. Let $g=f+x_{3}^{2} x_{1}^{2}$ be the second ray sum of $f$ with respect to $\alpha_{1}^{3}$ and $h=g+x_{4}^{2} x_{3}$ be the second ray sum of $g$ with respect to $\alpha_{3} \alpha_{1}^{2}$. Then the apolar algebra of

$$
h=x_{1}^{5}+x_{2}^{4}+x_{3}^{2} x_{1}^{2}+x_{4}^{2} x_{3}
$$

is smoothable and not obstructed. It has Hilbert function $(1,4,4,3,1,1)$.

Remark 5.26. The assumption $\operatorname{deg} \partial\lrcorner f>0$ in Proposition 5.23 is necessary: the polynomial $h=x_{1} x_{2} x_{3}+x_{4}^{2}+x_{5}^{2} x_{4}$ is obstructed, with length 12 and tangent space dimension $67>12.5$ over $k=\mathbb{C}$. The polynomial $g$ is the fourth ray sum of $x_{1} x_{2} x_{3}$ with respect to $\alpha_{1} \alpha_{2} \alpha_{3}$ and $h$ is the second ray sum of $g=x_{1} x_{2} x_{3}+x_{4}^{2}$ with respect to $\alpha_{4}$; thus this example satisfies the assumptions of Proposition 5.23 except for $\operatorname{deg} \partial\lrcorner f>0$. Note that in this case $\left.\alpha_{4}^{2}\right\lrcorner g \neq 0$.

\section{Proof of the main theorem and comments on the degree 14 case}

6A. Preliminary results. Let $r \geq 1$ be a natural number and $V$ be a constructible subset of $P_{\leq s}$. Assume that the apolar algebra $\operatorname{Apolar}(f)$ has length $r$ for every closed point $f \in V$. We may construct the incidence scheme $\{(f, \operatorname{Apolar}(f))\} \rightarrow V$, which is a finite flat family over $V$, and thus we obtain a morphism from $V$ to the (punctual) Hilbert scheme of $r$ points on an appropriate $\mathbb{P}^{n}$. See [Jelisiejew 2014a, Proposition 4.39] for details.

Consider $f \in P_{\leq s}$. The apolar algebra of $f$ has length at most $r$ if and only if the matrix of partials $S_{\leq s} f$ has rank at most $r$. This is a closed condition, so we obtain the following remark: 
Remark 6.1. Let $s$ be a positive integer and $V \subseteq P_{\leq s}$ be a constructible subset. Then the set $U$, consisting of $f \in V$ such that the apolar algebra of $f$ has maximal length (among the elements of $V$ ), is open in $V$. In particular, if $V$ is irreducible then $U$ is also irreducible.

Example 6.2. Let $P_{\geq 4}=k\left[x_{1}, \ldots, x_{n}\right]_{\geq 4}$ be the space of polynomials that are sums of monomials of degree at least 4. Suppose that the set $V \subseteq P_{\geq 4}$ parameterizing algebras with fixed Hilbert function $H$ is irreducible. Then also the set $W$ of polynomials $f \in P$ such that $f_{\geq 4} \in V$ is irreducible. Let $e:=H(1)$ and suppose that the symmetric decomposition of $H$ has zero rows $\Delta_{s-3}=(0,0,0,0)$ and $\Delta_{s-2}=$ $(0,0,0)$, where $s=\operatorname{deg} f$. We claim that a general element of $W$ corresponds to an algebra $B$ with Hilbert function $H_{\max }=H+(0, n-e, n-e, 0)$. Indeed, since we may only vary the degree-three part of the polynomial, the function $H_{B}$ has the form $H+(0, a, a, 0)+(0, b, 0)$ for some $a, b$ such that $a+b \leq n-e$. Therefore, algebras with Hilbert function $H_{\max }$ are precisely the algebras of maximal possible length. Since $H_{\max }$ is attained for $f_{\geq 4}+x_{e+1}^{3}+\cdots+x_{n}^{3}$, the claim follows from Remark 6.1.

6B. Lemmas on Hilbert functions. In the following, $H_{A}$ denotes the Hilbert function of an algebra $A$.

Lemma 6.3. Suppose that $A$ is a local Artin Gorenstein algebra of socle degree $s \geq 3$ such that $\Delta_{A, s-2}=(0,0,0)$. Then len $A \geq 2\left(H_{A}(1)+1\right)$. Furthermore, equality occurs if and only if $s=3$.

Proof. Consider the symmetric decomposition $\Delta_{\bullet}=\Delta_{A, \bullet}$ of $H_{A}$. From symmetry we have $\sum_{j} \Delta_{0}(j) \geq 2+2 \Delta_{0}(1)$, with equality only if $\Delta_{0}$ has no terms between 1 and $s-1$, i.e., when $s=3$. Similarly, $\sum_{j} \Delta_{i}(j) \geq 2 \Delta_{i}(1)$ for all $1 \leq i<s-2$. Summing these inequalities we obtain

$$
\text { len } A=\sum_{i<s-2} \sum_{j} \Delta_{i}(j) \geq 2+\sum_{i<s-2} 2 \Delta_{i}(1)=2+2 H_{A}(1) .
$$

Lemma 6.4. Let $A$ be a local Artin Gorenstein algebra of length at most 14. Suppose that $4 \leq H_{A}(1) \leq 5$. Then $H_{A}(2) \leq 5$.

Proof. Let $s$ be the socle degree of $A$. If $H_{A}(2) \geq 6$, then $H_{A}(3)+H_{A}(4)+\cdots \leq 3$; thus $s \in\{3,4,5\}$. The cases $s=3$ and $s=5$ immediately lead to contradiction - it is impossible to get the required symmetric decomposition. We will consider the case $s=4$. In this case, $H_{A}=(1, *, *, *, 1)$ and its symmetric decomposition is $(1, e, q, e, 1)+(0, m, m, 0)+(0, t, 0)$. Then $e=H_{A}(3) \leq 14-2-4-6=2$. Since $H_{A}(1)<H_{A}(2)$ we have $e<q$. This can only happen if $e=2$ and $q=3$. But then $14 \geq \operatorname{len} A=9+2 m+t$; thus $m \leq 2$ and $H_{A}(2)=m+q \leq 5$, a contradiction. 
Lemma 6.5. There does not exist a local Artin Gorenstein algebra with Hilbert function

$$
(1,4,3,4,1, \ldots, 1) .
$$

Proof. See [Iarrobino 1994, pp. 99-100] for the proof or [Casnati et al. 2014, Lemma 5.3] for a generalization. We provide a sketch for completeness. Suppose such an algebra $A$ exists and fix its dual socle generator $f \in k\left[x_{1}, \ldots, x_{4}\right]_{s}$ in the standard form. Let $I=\operatorname{ann}_{S}(f)$. The proof relies on two observations. First, the leading term of $f$ is, up to a constant, equal to $x_{1}^{s}$ and in fact we may take $f=x_{1}^{s}+f_{\leq 4}$. Moreover, from the symmetric decomposition it follows that the Hilbert functions of Apolar $\left(x_{1}^{s}+f_{4}\right)$ and Apolar $(f)$ are equal. Second, $h(3)=4=$ $3^{\langle 2\rangle}=h(2)^{\langle 2\rangle}$ is the maximal growth, so arguing similarly as in Lemma 2.9 we may assume that the degree-two part, $I_{2}$, of the ideal of gr $A$ is equal to $\left(\left(\alpha_{3}, \alpha_{4}\right) S\right)_{2}$. Then any derivative of $\left.\alpha_{3}\right\lrcorner f_{4}$ is a derivative of $x_{1}^{s}$, so a power of $x_{1}$. It follows that $\left.\alpha_{3}\right\lrcorner f_{4}$ itself is a power of $x_{1}$; similarly, $\left.\alpha_{4}\right\lrcorner f_{4}$ is a power of $x_{1}$. It follows that $f_{4} \in x_{1}^{3} \cdot k\left[x_{1}, x_{2}, x_{3}, x_{4}\right]+k\left[x_{1}, x_{2}\right]$, but then $f_{4}$ is annihilated by a linear form, which contradicts the fact that $f$ is in the standard form.

The following lemmas essentially deal with the limit-reducibility in the case $(1,4,4,3,1,1)$. Here the method is straightforward, but the cost is that the proof is broken into several cases and quite long.

Lemma 6.6. Let $f=x_{1}^{5}+f_{4}$ be a polynomial such that $H_{\text {Apolar }(f)}(2)<H_{\text {Apolar }\left(f_{4}\right)}(2)$. Let $\mathcal{Q}=S_{2} \cap \operatorname{ann}_{S}\left(x_{1}^{5}\right) \subseteq S_{2}$. Then $x_{1}^{2} \in \mathcal{Q} f_{4}$ and $\operatorname{ann}_{S}\left(f_{4}\right)_{2} \subseteq \mathcal{Q}$.

Proof. Note that $\operatorname{dim} \mathcal{Q} f_{4} \geq \operatorname{dim} S_{2} f_{4}-1=H_{\text {Apolar }\left(f_{4}\right)}(2)-1$. If $\operatorname{ann}_{S}\left(f_{4}\right)_{2} \nsubseteq \mathcal{Q}$, then there is a $q \in \mathcal{Q}$ such that $\alpha_{1}^{2}-q \in \operatorname{ann}_{S}\left(f_{4}\right)$. Then $\mathcal{Q} f_{4}=S_{2} f_{4}$ and we obtain a contradiction. Suppose that $x_{1}^{2} \notin \mathcal{Q} f_{4}$. Then the degree-two partials of $f$ contain a direct sum of $k x_{1}^{2}$ and $\mathcal{Q} f_{4}$, so they are at least $H_{\text {Apolar }\left(f_{4}\right)}(2)$-dimensional, so that $H_{\text {Apolar }(f)}(2) \geq H_{\text {Apolar }\left(f_{4}\right)}(2)$, a contradiction.

Lemma 6.7. Let $f=x_{1}^{5}+f_{4} \in P$ be a polynomial with $H_{\text {Apolar }(f)}=(1,3,3,3,1,1)$ and $H_{\mathrm{Apolar}\left(f_{4}\right)}=(1,3,4,3,1)$. Suppose that $\left.\alpha_{1}^{3}\right\lrcorner f_{4}=0$ and that $\left(\operatorname{ann}_{S}\left(f_{4}\right)\right)_{2}$ defines a complete intersection. Then Apolar $\left(f_{4}\right)$ and $\operatorname{Apolar}(f)$ are complete intersections.

Proof. Let $I:=\operatorname{ann}_{S}\left(f_{4}\right)$. First we will prove that $\operatorname{ann}_{S}\left(f_{4}\right)=\left(q_{1}, q_{2}, c\right)$, where $\left\langle q_{1}, q_{2}\right\rangle=I_{2}$ and $c \in I_{3}$. Then, of course, Apolar $\left(f_{4}\right)$ is a complete intersection. By assumption, $q_{1}$ and $q_{2}$ form a regular sequence. Thus there are no syzygies of degree at most three in the minimal resolution of Apolar $\left(f_{4}\right)$. By the symmetry of the minimal resolution - see [Eisenbud 1995, Corollary 21.16] — there are no generators of degree at least four in the minimal generating set of $I$. Thus $I$ is generated in degrees two and three. But $H_{S /\left(q_{1}, q_{2}\right)}(3)=4=H_{S / I}(3)+1$; thus there 
is a cubic $c$ such that $I_{3}=k c \oplus\left(q_{1}, q_{2}\right)_{3}$, then $\left(q_{1}, q_{2}, c\right)=I$, so Apolar $\left(f_{4}\right)=S / I$ is a complete intersection.

Let $\mathcal{Q}:=\operatorname{ann}_{S}\left(x_{1}^{5}\right) \cap S_{2} \subseteq S_{2}$. By Lemma 6.6 we have $q_{1}, q_{2} \in \mathcal{Q}$, so that $\alpha_{1}^{3} \in I \backslash\left(q_{1}, q_{2}\right)$, then $I=\left(q_{1}, q_{2}, \alpha_{1}^{3}\right)$. Moreover, by the same lemma, there exists $\sigma \in \mathcal{Q}$ such that $\sigma\lrcorner f_{4}=x_{1}^{2}$.

Now we prove Apolar $(f)$ is a complete intersection. Let $J:=\left(q_{1}, q_{2}, \alpha_{1}^{3}-\sigma\right) \subseteq$ $\operatorname{ann}_{S}(f)$. We will prove that $S / J$ is a complete intersection. Since $q_{1}, q_{2}, \alpha_{1}^{3}$ is a regular sequence, the set $S /\left(q_{1}, q_{2}\right)$ is a cone over a scheme of dimension zero and $\alpha_{1}^{3}$ does not vanish identically on any of its components. Since $\sigma$ has degree two, $\alpha_{1}^{3}-\sigma$ also does not vanish identically on any of the components of Spec $S /\left(q_{1}, q_{2}\right)$; thus Spec $S / J$ has dimension zero, so it is a complete intersection (see also [Valabrega and Valla 1978, Corollary 2.4, Remark 2.5]). Then the quotient by $J$ has length at most $\operatorname{deg}\left(q_{1}\right) \operatorname{deg}\left(q_{2}\right) \operatorname{deg}\left(\alpha_{1}^{3}-\sigma\right)=12=\operatorname{dim}_{k} S / \operatorname{ann}_{S}(f)$. Since $J \subseteq \operatorname{ann}_{S}(f)$, we have $\operatorname{ann}_{S}(f)=J$ and Apolar $(f)$ is a complete intersection.

Lemma 6.8. Let $f=x_{1}^{5}+f_{4}+g$, where $\operatorname{deg} g \leq 3$, be a polynomial such that $H_{\mathrm{Apolar}\left(f_{\geq 4}\right)}=(1,3,3,3,1,1)$ and $H_{\mathrm{Apolar}\left(f_{4}\right)}=(1,3,4,3,1)$. Suppose that $\left.\alpha_{1}^{3}\right\lrcorner f_{4}=0$ and that $\left(\operatorname{ann}_{S}\left(f_{4}\right)\right)_{2}$ does not define a complete intersection. Then Apolar $(f)$ is limit-reducible.

Proof. Let $\left\langle q_{1}, q_{2}\right\rangle=\left(\operatorname{ann}_{S}\left(f_{4}\right)\right)_{2}$. Since $q_{1}, q_{2}$ do not form a regular sequence, we have, after a linear transformation $\varphi$, two possibilities: $q_{1}=\alpha_{1} \alpha_{2}$ and $q_{2}=\alpha_{1} \alpha_{3}$, or $q_{1}=\alpha_{1}^{2}$ and $q_{2}=\alpha_{1} \alpha_{2}$. Let $\beta$ be the image of $\alpha_{1}$ under $\varphi$, so that $\left.\beta^{3}\right\lrcorner f_{4}=0$.

Suppose first that $q_{1}=\alpha_{1} \alpha_{2}$ and $q_{2}=\alpha_{1} \alpha_{3}$. If $\beta$ is, up to a constant, equal to $\alpha_{1}$, then $\alpha_{1} \alpha_{2}, \alpha_{1} \alpha_{3}, \alpha_{1}^{3} \in \operatorname{ann}_{S}\left(f_{4}\right)$, so that $\alpha_{1}^{2}$ is in the socle of $\operatorname{Apolar}\left(f_{4}\right)$, a contradiction. Thus we may assume, after another change of variables, that $\beta=\alpha_{2}$, $q_{1}=\alpha_{1} \alpha_{2}$ and $q_{2}=\alpha_{1} \alpha_{3}$. Then $f=x_{2}^{5}+f_{4}+\hat{g}=x_{2}^{5}+x_{1}^{4}+\hat{h}+\hat{g}$, where $\hat{h} \in k\left[x_{1}, x_{3}\right]$ and $\operatorname{deg}(\hat{g}) \leq 3$. Then, by Lemma 4.2, we may assume that $\left.\alpha_{1}^{2}\right\lrcorner f=0$, so Apolar $(f)$ is limit-reducible by Proposition 5.13. See also Example 5.14 (the degree assumption in the example can easily be modified).

Suppose now that $q_{1}=\alpha_{1}^{2}$ and $q_{2}=\alpha_{1} \alpha_{2}$. If $\beta$ is not a linear combination of $\alpha_{1}$ and $\alpha_{2}$, then we may assume $\beta=\alpha_{3}$. Let $m$ in $f_{4}$ be any monomial divisible by $x_{1}$. Since $q_{1}, q_{2} \in \operatorname{ann}_{S}\left(f_{4}\right)$, we see that $m=\lambda x_{1} x_{3}^{3}$ for some $\lambda \in k$. But, since $\beta^{3} \in \operatorname{ann}_{S}\left(f_{4}\right)$, we have $m=0$. Thus $f_{4}$ does not contain $x_{1}$, so $H_{\text {Apolar }\left(f_{4}\right)}(1)<3$, a contradiction. Thus $\beta \in\left\langle\alpha_{1}, \alpha_{2}\right\rangle$. Suppose $\beta=\lambda \alpha_{1}$ for some $\lambda \in k \backslash\{0\}$. Applying Lemma 6.6 to $f_{\geq 4}$ we see that $x_{1}^{2}$ is a derivative of $f_{4}$, so $\left.\beta^{2}\right\lrcorner f_{4} \neq 0$, but $\left.\left.\beta^{2}\right\lrcorner f_{4}=\lambda^{2} q_{1}\right\lrcorner f_{4}=0$, a contradiction. Thus $\beta=\lambda_{1} \alpha_{1}+\lambda_{2} \alpha_{2}$ and, changing $\alpha_{2}$, we may assume that $\beta=\alpha_{2}$. This substitution does not change $\left\langle\alpha_{1}^{2}, \alpha_{1} \alpha_{2}\right\rangle$. Now we directly check that $f_{4}=x_{3}^{2}\left(\kappa_{1} x_{1} x_{3}+\kappa_{2} x_{2}^{2}+\kappa_{3} x_{2} x_{3}+\kappa_{4} x_{3}^{2}\right)$ for some $\kappa_{\bullet} \in k$. Since $x_{1}$ is a derivative of $f$, we have $\kappa_{1} \neq 0$. Then a nonzero element $\kappa_{2} \alpha_{1} \alpha_{3}-\kappa_{1} \alpha_{2}^{2}$ annihilates $f_{4}$, a contradiction with $H_{\text {Apolar }\left(f_{4}\right)}(2)=4$. 
Lemma 6.9. Let a quartic $f_{4}$ be such that $H_{\mathrm{Apolar}\left(f_{4}\right)}=(1,3,3,3,1)$ and $\left.\alpha_{1}^{3}\right\lrcorner f_{4}=0$. Then $H_{\text {Apolar }\left(x_{1}^{5}+f_{4}\right)}(2) \geq 4$.

Proof. Let $\mathcal{Q}=\operatorname{ann}_{S}\left(x_{1}^{5}\right)_{2} \subseteq S_{2}$. Let $I$ denote the apolar ideal of $f_{4}$. By Proposition 4.9 we see that $I$ is minimally generated by three elements of degree two and two elements of degree four. In particular, there are no cubics in the generating set. Since $\alpha_{1}^{3} \in I_{3}$, there is an element in $\sigma \in I_{2}$ such that $\sigma=\alpha_{1}^{2}-q$, where $q \in \mathcal{Q}$. Therefore, $\left.\mathcal{Q}\lrcorner f_{4}=S_{2}\right\lrcorner f_{4}$. Moreover, $\sigma$ does not annihilate $x_{1}^{2}$, so that $x_{1}^{2}$ is not a partial of $f_{4}$. We see that $x_{1}^{2}$ and $\left.\mathcal{Q}\right\lrcorner f_{4}$ are leading forms of partials of $x_{1}^{5}+f_{4}$; thus

$$
\left.\left.H_{\text {Apolar }\left(x_{1}^{5}+f_{4}\right)}(2) \geq 1+\operatorname{dim}(\mathcal{Q}\lrcorner f_{4}\right)=1+\operatorname{dim}\left(S_{2}\right\lrcorner f_{4}\right)=1+H_{\text {Apolar }\left(f_{4}\right)}(2)=4 .
$$

Remark 6.10. Given the assumptions of Lemma 6.9, it is not hard to deduce that $H_{\text {Apolar }\left(x_{1}^{5}+f_{4}\right)}=(1,3,4,3,1,1)$ by analyzing the possible symmetric decompositions. We do not need this stronger statement, so we omit the proof.

6C. Proofs. The following proposition generalizes results about algebras with Hilbert function $(1,5,5,1)$ obtained in [Jelisiejew 2014b; Bertone et al. 2012].

Proposition 6.11. Let A be a local Artin Gorenstein algebra of socle degree three and $H_{A}(2) \leq 5$. Then $A$ is smoothable.

Proof. Suppose that the Hilbert function of $A$ is $(1, n, e, 1)$. By Proposition 4.5 the dual socle generator of $A$ may be put in the form $f+x_{e+1}^{2}+\cdots+x_{n}^{2}$, where $f \in k\left[x_{1}, \ldots, x_{e}\right]$. By repeated use of Example 5.12 we see that $A$ is a limit of algebras of the form Apolar $(f) \times k^{\oplus n-e}$. Thus it is smoothable if and only if $B=$ Apolar $(f)$ is.

Let $e:=H_{A}(2)$; then $H_{B}=(1, e, e, 1)$. If $H_{B}(1)=e \leq 3$, then $B$ is smoothable. It remains to consider $4 \leq e \leq 5$. The set of points corresponding to algebras with Hilbert function $(1, e, e, 1)$ is irreducible in $\mathcal{H i l b}_{2 e+2}\left(\mathbb{P}^{e}\right)$, by Remark 6.1 for the obvious parameterization (as mentioned in [Iarrobino 1984, Theorem I, p. 350]); thus, it will be enough to find a smooth point in this set which corresponds to a smoothable algebra. The cases $e=4$ and $e=5$ are considered in Examples 5.22 and 5.24, respectively.

Remark 6.12. The claim of Proposition 6.11 holds true if we replace the assumption $H_{A}(2) \leq 5$ by $H_{A}(2)=7$, thanks to the smoothability of local Artin Gorenstein algebras with Hilbert function $(1,7,7,1)$; see [Bertone et al. 2012]. We will not use this result.

Lemma 6.13. Let $A$ be a local Artin Gorenstein algebra with Hilbert function $H_{A}$ beginning with $H_{A}(0)=1, H_{A}(1)=4, H_{A}(2)=5, H_{A}(3) \leq 2$. Then $A$ is smoothable. 
Proof. Let $f$ be a dual socle generator of $A$ in the standard form. It follows from Macaulay's growth theorem that $H_{A}(m) \leq 2$ for all $m \geq 3$, so that $H_{A}=$ $(1,4,5,2,2, \ldots, 2,1, \ldots, 1)$. Let $s$ be the socle degree of $A$.

Let $\Delta_{A, s-2}=(0, q, 0)$ be the $(s-2)$-nd row of the symmetric decomposition of $H_{A}$. If $q>0$, then by Example 5.12 we know that $A$ is limit-reducible; it is a limit of algebras of the form $B \times k$ such that $H_{B}(1)=H_{A}(1)-1=3$. Then the algebra $B$ is smoothable (see [Casnati and Notari 2009, Proposition 2.5]), so $A$ is also smoothable. In the following we assume that $q=0$.

We claim that $f_{\geq 4} \in k\left[x_{1}, x_{2}\right]$. Indeed, the symmetric decomposition of the Hilbert function is either $(1,1, \ldots, 1)+(0,1, \ldots, 1,0)+(0,0,1,0,0)+(0,2,2,0)$ or $(1,2, \ldots, 2,1)+(0,0,1,0,0)+(0,2,2,0)$. Thus, $e(s-3)=\sum_{i \geq 3} \Delta_{i}(1)=2$, so that $f_{\geq 4} \in k\left[x_{1}, x_{2}\right]$ and $H_{\text {Apolar }\left(f_{\geq 4}\right)}(1)=2$; in particular, $x_{1}$ is a derivative of $f_{\geq 4}$, i.e., there exists a $\partial \in S$ such that $\partial\lrcorner f_{\geq 4}=x_{1}$. Then we may assume $\partial \in \mathfrak{m}_{S}^{3}$, so $\left.\partial^{2}\right\lrcorner f=0$.

Let us fix $f_{\geq 4}$ and consider the set of all polynomials of the form $h=f_{\geq 4}+g$, where $g \in k\left[x_{1}, x_{2}, x_{3}, x_{4}\right]$ has degree at most three. By Example 6.2 the apolar algebra of a general such polynomial will have Hilbert function $H_{A}$. The set of polynomials $h$ with fixed $h_{\geq 4}=f_{\geq 4}$ such that $H_{\text {Apolar }(h)}=H_{A}$ is irreducible. This set contains $h:=f_{\geq 4}+x_{3}^{2} x_{1}+x_{4}^{2} x_{3}$. To finish the proof is it enough to show that $h$ is smoothable and unobstructed. Since $\operatorname{Apolar}\left(f_{\geq 4}\right)$ is a complete intersection, this follows from Example 5.24.

The following Theorem 6.14 generalizes numerous earlier smoothability results on stretched (by Sally, [1979]), 2-stretched (by Casnati and Notari [2014a]) and almost-stretched (by Elias and Valla [2011]) algebras. It is important to understand that, in contrast with the mentioned papers, we avoid a full classification of algebras. In the course of the proof we give some partial classification.

Theorem 6.14. Let $A$ be a local Artin Gorenstein algebra with Hilbert function $H_{A}$ satisfying $H_{A}(2) \leq 5$ and $H_{A}(3) \leq 2$. Then $A$ is smoothable.

Proof. We proceed by induction on len $A$, the case len $A=1$ being trivial. If $A$ has socle degree three, then the result follows from Proposition 6.11. Suppose that $A$ has socle degree $s \geq 4$.

Let $f$ be a dual socle generator of $A$ in the standard form. If the symmetric decomposition of $H_{A}$ has a term $\Delta_{s-2}=(0, q, 0)$ with $q \neq 0$, then by Example 5.12, we have that $A$ is a limit of algebras of the form $B \times k$, where $B$ satisfies the assumptions $H_{B}(2) \leq 5$ and $H_{B}(2) \leq 2$ on the Hilbert function. Then $B$ is smoothable by induction, so also $A$ is smoothable. Further in the proof we assume that $\Delta_{A, s-2}=(0,0,0)$.

We would like to understand the symmetric decomposition of the Hilbert function $H_{A}$ of $A$. Since $H_{A}$ satisfies the Macaulay growth condition (see Section 2E) it 
follows that $H_{A}=(1, n, m, 2,2, \ldots, 2,1, \ldots, 1)$, where the number of twos is possibly zero. If follows that the possible symmetric decompositions of the Hilbert function are:

(1) $(1,2,2, \ldots, 2,1)+(0,0,1,0,0)+(0, n-3, n-3,0)$,

(2) $(1,1,1 \ldots, 1,1)+(0,1,1, \ldots, 1,0)+(0,0,1,0,0)+(0, n-3, n-3,0)$,

(3) $(1,1,1 \ldots, 1,1)+(0,1,2,1,0)+(0, n-3, n-3,0)$,

(4) $(1, \ldots, 1)+(0, n-1, n-1,0)$,

(5) $(1,2, \ldots, 2,1)+(0, n-2, n-2,0)$,

(6) $(1, \ldots, 1)+(0,1, \ldots, 1,0)+(0, n-2, n-2,0)$,

and that the decomposition is uniquely determined by the Hilbert function. In all cases we have $H_{A}(1) \leq H_{A}(2) \leq 5$, so $f \in k\left[x_{1}, \ldots, x_{5}\right]$. Let us analyze the first three cases. In each of them we have $H_{A}(2)=H_{A}(1)+1$. If $H_{A}(1) \leq 3$, then $A$ is smoothable; see [Casnati and Notari 2009, Corollary 2.4]. Suppose $H_{A}(1) \geq 4$. Since $H_{A}(2) \leq 5$, we have $H_{A}(2)=5$ and $H_{A}(1)=4$. In this case the result follows from Lemma 6.13 above.

It remains to analyze the three remaining cases. The proof is similar to the proof of Lemma 6.13, however here it essentially depends on induction. Let $f_{\geq 4}$ be the sum of homogeneous components of $f$ which have degree at least four. Since $f$ is in the standard form, we have $f_{\geq 4} \in k\left[x_{1}, x_{2}\right]$. The decomposition of the Hilbert function Apolar $\left(f_{\geq 4}\right)$ is one of the decompositions $(1, \ldots, 1)$, $(1,2 \ldots, 2,1)$ or $(1, \ldots, 1)+(0,1, \ldots, 1,0)$, depending on the decomposition of the Hilbert function of Apolar $(f)$.

Let us fix a vector $\hat{h}=(1,2,2,2, \ldots, 2,1,1, \ldots, 1)$ and take the sets $V_{1}:=\left\{f \in k\left[x_{1}, x_{2}\right] \mid H_{\text {Apolar }(f)}=\hat{h}\right\} \quad$ and $\quad V_{2}:=\left\{f \in k\left[x_{1}, \ldots, x_{n}\right] \mid f_{\geq 4} \in V_{1}\right\}$.

By Proposition 4.8 the set $V_{1}$ is irreducible and thus $V_{2}$ is also irreducible. The Hilbert function of the apolar algebra of a general member of $V_{2}$ is, by Example 6.2, equal to $H_{A}$. It remains to show that the apolar algebra of this general member is smoothable.

Proposition 4.8 implies that the general member of $V_{2}$ has (after a nonlinear change of coordinates) the form $f+\partial\lrcorner f$, where $f=x_{1}^{s}+x_{2}^{s_{2}}+g$ for some $g$ of degree at most three. Using Lemma 4.2 we may assume (after another nonlinear change of coordinates) that $\left.\alpha_{1}^{2}\right\lrcorner g=0$.

Let $B:=\operatorname{Apolar}\left(x_{1}^{s}+x_{2}^{s_{2}}+g\right)$. We will show that $B$ is smoothable. Since $s \geq 4=2 \cdot 2$, Proposition 5.13 shows that $B$ is limit-reducible. Analyzing the fibers of the resulting degeneration, as in Example 5.15, we see that they have the form $B^{\prime} \times k$, where $B^{\prime}=\operatorname{Apolar}(\hat{f})$ and $\hat{f}=\lambda^{-1} x_{1}^{s-1}+x_{2}^{s_{2}}+g$. Then $H_{B^{\prime}}(3)=H_{\text {Apolar }\left(\hat{f}_{\geq 4}\right)}(3) \leq 2$. Moreover, $\hat{f} \in k\left[x_{1}, \ldots, x_{5}\right]$, so that $H_{B^{\prime}}(1) \leq 5$. Now, analyzing the possible 
symmetric decompositions of $H_{B^{\prime}}$, which are listed above, we see that $H_{B^{\prime}}(2) \leq$ $H_{B^{\prime}}(1)=5$. It follows from induction on the length that $B^{\prime}$ is smoothable; thus $B^{\prime} \times k$ and $B$ are smoothable.

Proposition 6.15. Let A be a local Artin Gorenstein algebra of socle degree four satisfying len $A \leq 14$. Then $A$ is smoothable.

Proof. We proceed by induction on the length of $A$. Then, by Proposition 6.11 (and the fact that all algebras of socle degree at most two are smoothable), we may assume that all algebras of socle degree at most four and length less than len $A$ are smoothable.

If $\Delta_{A, 1}=(0, q, 0)$ with $q \neq 0$, then by Example 5.12 the algebra $A$ is a limit of algebras of the form $A^{\prime} \times k$, where $A^{\prime}$ has socle degree four. Hence $A$ is smoothable. Therefore we assume $q=0$. Then $H_{A}(1) \leq 5$ by Lemma 6.3. Moreover, we may assume $H_{A}(1) \geq 4$, since otherwise $A$ is smoothable by [Casnati and Notari 2009, Corollary 2.4].

The symmetric decomposition of $H_{A}$ is $(1, n, m, n, 1)+(0, p, p, 0)$ for some $n$, $m$ and $p$. By the fact that $n \leq 5$ and Stanley's result [1996, p. 67], we have $n \leq m$; thus $n \leq 4$ and $H_{A}(2) \leq H_{A}(1) \leq 5$. Due to len $A \leq 14$, we have four cases, $n=1,2,3,4$, and five possible shapes of Hilbert functions, $H_{A}=(1, *, *, 1,1)$, $H_{A}=(1, *, *, 2,1), H_{A}=(1,4,4,3,1), H_{A}=(1,4,4,4,1)$ or $H_{A}=(1,4,5,3,1)$.

The conclusion in the first two cases follows from Theorem 6.14. In the remaining cases we first look for a suitable irreducible set of dual socle generators parameterizing algebras with prescribed $H_{A}$. We examine the case $H_{A}=(1,4,4,3,1)$. Consider the set of $f \in P=k\left[x_{1}, x_{2}, x_{3}, x_{4}\right]$ in the standard form and such that the apolar algebra of $f$ has Hilbert function $H_{A}$. We claim that this set is irreducible. Since the leading form $f_{4}$ of such an $f$ has Hilbert function $(1,3,3,3,1)$, the set of possible leading forms is irreducible by Proposition 4.9. Then the irreducibility follows from Example 6.2. The irreducibility in the cases $H_{A}=(1,4,4,4,1)$ and $H_{A}=(1,4,5,3,1)$ follows similarly from Proposition 4.10 together with Example 6.2. In the first two cases we see that $f_{4}$ is a sum of powers of variables; then Example 5.14 shows that the apolar algebra $A$ of a general $f$ is limit-reducible. More precisely, $A$ is a limit of algebras of the form $A^{\prime} \times k$, where $A^{\prime}$ has socle degree at most four (compare Example 5.15). Then $A$ is smoothable. In the last case, Example 5.21 gives an unobstructed algebra in this irreducible set. This completes the proof.

Lemma 6.16. Let $A$ be a local Artin Gorenstein algebra with Hilbert function $(1,4,4,3,1,1)$. Then A is limit-reducible.

Proof. Let $s=5$ be the socle degree of $A$. If $\Delta_{A, s-2} \neq(0,0,0)$ then $A$ is limitreducible by Example 5.12, so we assume $\Delta_{A, s-2}=(0,0,0)$. The only possible 
symmetric decomposition of the Hilbert function $H_{A}$ with $\Delta_{A, s-2}=(0,0,0)$ is

$$
(1,4,4,3,1,1)=(1,1,1,1,1,1)+(0,2,2,2,0)+(0,1,1,0) .
$$

Let us take a dual socle generator $f$ of $A$. We assume that $f$ is in the standard form $f=x_{1}^{5}+f_{4}+g$, where $\operatorname{deg} g \leq 3$. Then $H_{\text {Apolar }\left(x_{1}^{5}+f_{4}\right)}=(1,3,3,3,1,1)$. We analyze the possible Hilbert functions of $B=\operatorname{Apolar}\left(f_{4}\right)$. By Lemma 4.2 we may assume that $\left.\alpha_{1}^{3}\right\lrcorner f_{4}=0$. Suppose first that $H_{B}(1) \leq 2$. From (12) it follows that $H_{\text {Apolar }\left(x_{1}^{5}+f_{4}\right)}(1)=3$, so that $H_{B}(1)=2$ and we may assume that $f_{4} \in k\left[x_{2}, x_{3}\right]$. Then, by Lemma 4.2, we may further assume $\left.\alpha_{1}^{2}\right\lrcorner\left(f-x_{1}^{5}\right)=0$; then Proposition 5.13 asserts that $A=\operatorname{Apolar}(f)$ is limit-reducible.

Suppose now that $H_{B}(1)=3$. Since $x_{1}^{5}$ is annihilated by a codimensionone space of quadrics, we have $H_{B}(2) \leq H_{A}(2)+1$, so there are two possibilities: $H_{B}=(1,3,3,3,1)$ or $H_{B}=(1,3,4,3,1)$. By Lemma 6.9 the case $H_{B}=(1,3,3,3,1)$ is not possible, so that $H_{B}=(1,3,4,3,1)$. Now, by Lemma 6.8 , we may consider only the case when $\left(\operatorname{ann}_{S}\left(f_{4}\right)\right)_{2}$ is a complete intersection; then, by Lemma 6.7, we have that $\operatorname{Apolar}\left(x_{1}^{5}+f_{4}\right)$ is a complete intersection. In this case we will actually prove that $A$ is smoothable.

By Example 6.2, the set $W$ of polynomials $f$ with fixed leading polynomial $f_{\geq 4}$ and Hilbert function $H_{\operatorname{Apolar}(f)}=(1,4,4,3,1,1)$ is irreducible. Consider the apolar algebra $B$ of the polynomial $x_{1}^{5}+f_{4}+x_{4}^{2} x_{1} \in W$. By Proposition 5.10, this algebra is the limit of smoothable algebras $\operatorname{Apolar}\left(x_{1}^{5}+f_{4}\right) \times \operatorname{Apolar}\left(x_{1}\right)$, so it is smoothable. By Corollary 5.20, the algebra $B$ is unobstructed. Thus the apolar algebra of every element of $W$ is smoothable; in particular $A$ is smoothable.

Now we are ready to prove Theorem 6.17 , which is the algebraic counterpart of Theorems A and B.

Theorem 6.17. Let $A$ be an Artin Gorenstein algebra of length at most 14. Then either $A$ is smoothable or it is local with Hilbert function $(1,6,6,1)$. In particular, if $A$ has length at most 13 , then $A$ is smoothable.

Proof. By the discussion in Section 2D it is enough to consider local algebras. Let $A$ be a local algebra of length at most 14 and of socle degree $s$. By $H$ we denote the Hilbert function of $A$. As mentioned in Section 2D it is enough to prove $A$ is limit-reducible. On the contrary, suppose that $A$ is strongly nonsmoothable in the sense of Definition 2.5. By Example 5.12 we have $\Delta_{A, s-2}=(0,0,0)$. Then, by Lemma 6.3 , we see that either $H=(1,6,6,1)$ or $H(1) \leq 5$. It is enough to consider $H(1) \leq 5$. If $s=3$ then $H(2) \leq H(1) \leq 5$, so by Proposition 6.11 we may assume $s>3$. By Proposition 6.15 it follows that we may consider only $s \geq 5$.

If $H(1) \leq 3$ then $A$ is smoothable by [Casnati and Notari 2009, Corollary 2.4]; thus we may assume $H(1) \geq 4$. By Lemma 6.4 we see that $H(2) \leq 5$. Then, by Theorem 6.14, we may reduce to the case $H(3) \geq 3$. By Macaulay's growth theorem 
we have $H(2) \geq 3$. Then $\sum_{i>3} H(i) \leq 14-11$, so we are left with several possibilities: $H=(1,4,3,3,1,1,1), H=(1,4,3,3,2,1)$ or $H=(1, *, *, *, 1,1)$. In the first two cases it follows from the symmetric decomposition that $\Delta_{A, s-2} \neq(0,0,0)$, which is a contradiction. We examine the last case. By Lemma 6.5 there does not exist an algebra with Hilbert function $(1,4,3,4,1,1)$. Thus the only possibilities are $(1,4,3,3,1,1),(1,5,3,3,1,1)$ and $(1,4,4,3,1,1)$. Once more, it can be checked directly that in the first two cases $\Delta_{A, s-2} \neq(0,0,0)$. The last case is the content of Lemma 6.16.

Remark 6.18. Assume char $k=0$. Iarrobino and Emsalem [1978] analyzed the tangent space to the Hilbert scheme. Iarrobino and Kanev [1999, Lemma 6.21] claim that using Macaulay they are able to check that the tangent space to $\mathcal{H i l b}_{6}\left(\mathbb{P}^{14}\right)$ has dimension 76 at a point corresponding to a general local Gorenstein algebra $A$ with Hilbert function $(1,6,6,1)$; see [Casnati and Notari 2011] for further details. Since $76<(1+6+6+1) \cdot 6$ this shows that $A$ is nonsmoothable. Moreover, since all algebras of degree at most 13 are smoothable, $A$ is strongly nonsmoothable.

To prove Theorem B, we need to show that the nonsmoothable part of $\mathcal{H} i l b_{14}^{G} \mathbb{P}^{n}$ (for $n \geq 6$ ) is irreducible. The algebraic version of (a generalization of) this statement is the following lemma:

Lemma 6.19. Let $n \geq m$ be natural numbers and $V \subseteq P_{\leq 3}=k\left[x_{1}, \ldots, x_{n}\right]_{\leq 3}$ be the set of $f \in P$ such that $H_{\mathrm{Apolar}(f)}=(1, m, m, 1)$. Then $V$ is constructible and irreducible.

Proof. Let $V_{\mathrm{gr}}=V \cap P_{3}$ denote the set of graded algebras with Hilbert function $(1, m, m, 1)$. This is a constructible subset of $P_{3}$. To an element $f_{3} \in V_{\mathrm{gr}}$ we may associate the tangent space to Apolar $\left(f_{3}\right)$, which is isomorphic to $\left.S_{2}\right\lrcorner f_{3}$. We define

$$
\left.\left\{\left(f_{3},[W]\right) \in V_{\mathrm{gr}} \times \operatorname{Gr}(m, n) \mid W \supseteq S_{2}\right\lrcorner f_{3}\right\},
$$

which is an open subset in a vector bundle $\left.\left\{\left(f_{3},[W]\right) \in P_{3} \times \operatorname{Gr}(m, n) \mid W \supseteq S_{2}\right\lrcorner f_{3}\right\}$ over $\operatorname{Gr}(m, n)$, given by the condition $\left.\operatorname{dim} S_{2}\right\lrcorner f_{3} \geq m$. Let $f \in V$ and write it as $f=f_{3}+f_{\leq 2}$, where $\operatorname{deg} f_{\leq 2} \leq 2$. Then $H_{\text {Apolar }\left(f_{3}\right)}=(1, m, m, 1)$. Therefore, we obtain a morphism $\varphi: V \rightarrow V_{\mathrm{gr}}$ sending $f$ to $f_{3}$. We will analyze its fibers. Let $f_{3} \in V_{\mathrm{gr}}$ and $f=f_{3}+f_{\leq 2} \in P_{\leq 3}$, where $\operatorname{deg} f_{\leq 2} \leq 2$. Then $H_{\text {Apolar }(f)}=(1, M, m, 1)$ for some $M \geq m$. Moreover, $M=m$ if and only if $\alpha\lrcorner f_{\leq 2}$ is a partial of $f_{3}$ for every $\alpha$ annihilating $f_{3}$. The fiber of $\varphi$ over $f$ is an affine subspace of $P_{\leq 2}$ defined by these conditions and the morphism, writing $f=f_{3}+f_{\leq 2}$,

$$
\left.\left.\left\{(f,[W]) \in V \times \operatorname{Gr}(m, n) \mid W \supseteq S_{2}\right\lrcorner f_{3}\right\} \rightarrow\left\{\left(f_{3},[W]\right) \in V_{\mathrm{gr}} \times \operatorname{Gr}(m, n) \mid W \supseteq S_{2}\right\lrcorner f_{3}\right\}
$$

is a projection from a vector bundle, which is thus irreducible. Since $V$ admits a surjection from this bundle, it is irreducible as well. Moreover, the above shows that $V$ is constructible. 
Proof of Theorems A and B. The locus of points of the Hilbert scheme corresponding to smooth (i.e., reduced) algebras of length $d$ is irreducible, as an image of an open subset of the $d$-symmetric product of $\mathbb{P}^{n}$, and smooth. The locus of points corresponding to smoothable algebras is the closure of the aforementioned locus, so it is also irreducible. If $d \leq 13$, or $d \leq 14$ and $n \leq 5$, this locus is the whole Hilbert scheme by Theorem 6.17, and the claim follows.

Now consider the case $d=14$ and $n \geq 6$. Let $\mathcal{V}$ be the set of points of the Hilbert scheme corresponding to local Gorenstein algebras with Hilbert function $(1,6,6,1)$. By Remark 6.18 these are the only nonsmoothable algebras of length 14; thus they deform only to local algebras with the same Hilbert function. Therefore, $\mathcal{V}$ is a sum of irreducible components of the Hilbert scheme. We will prove that $\mathcal{V}$ is an irreducible set whose general point is smooth.

Let $\mathcal{V}_{p} \subseteq \mathcal{V}$ denote the set consisting of schemes supported at a fixed point $p \in \mathbb{P}^{n}$. Then $\mathcal{V}$ is dominated by a set $\mathcal{V}_{p} \times \mathbb{P}^{n}$. Note that an irreducible scheme supported at a point $p$ may be identified with a Gorenstein quotient of the power series ring having Hilbert function $(1,6,6,1)$. These quotients are parameterized by the dual generators. More precisely, the set of $V$ of $f \in k\left[x_{1}, \ldots, x_{n}\right]_{\leq 3}$ such that $H_{\text {Apolar }(f)}=(1,6,6,1)$ gives a morphism

$$
V \rightarrow \mathcal{V}_{p} \subseteq \mathcal{H i l b} b_{14}^{G} \mathbb{P}^{n}
$$

which sends $f$ to $\operatorname{Spec} \operatorname{Apolar}(f)$ supported at $p$ (see Section 6A). Since $V \rightarrow \mathcal{V}_{p}$ is surjective and $V$ is irreducible by Lemma 6.19, we see that $\mathcal{V}_{p}$ is irreducible. Then $\mathcal{V}$ is irreducible as well.

Take a smooth point of $\mathcal{H} i l b_{14}^{G} \mathbb{P}^{6}$ which corresponds to an algebra $A$ with Hilbert function $(1,6,6,1)$. Then any point of $\mathcal{H} i b_{14}^{G} \mathbb{P}^{n}$ corresponding to an embedding $\operatorname{Spec} A \subseteq \mathbb{P}^{n}$ is smooth, by [Casnati and Notari 2009, Lemma 2.3]. This concludes the proof.

\section{List of symbols}

All symbols appearing below are defined in Section 2.

- $k$ is an algebraically closed field of characteristic $\neq 2,3$.

- $P=k\left[x_{1}, \ldots, x_{n}\right]$ is a polynomial ring in $n$ variables and fixed basis.

- $S=k \llbracket \alpha_{1}, \ldots, \alpha_{n} \rrbracket$ is a power series ring dual (see Section $2 \mathrm{~B}$ ) to $P$, with a fixed (dual) basis.

- $\mathfrak{m}_{S}$ is the maximal ideal of $S$.

- $S_{\text {poly }}=k\left[\alpha_{1}, \ldots, \alpha_{n}\right]$ is a polynomial subring of $S$ defined by the choice of the basis.

- $H_{A}$ is the Hilbert function of a local Artin algebra $A$. 
- $\Delta_{A, i}, \Delta_{i}$ is the $i$-th row of the symmetric decomposition of the Hilbert function of a local Artin Gorenstein algebra $A$; see Theorem 2.3.

- $e(a)$ is the $a$-th "embedding dimension", which is equal to $\sum_{t=0}^{a} \Delta_{t}(1)$; see Definition 3.1.

- $\operatorname{ann}_{S}(f)$ is the annihilator of $f \in P$ with respect to the action of $S$.

- Apolar $(f)$ is the apolar algebra of $f \in P$, equal to $S / \operatorname{ann}_{S}(f)$.

\section{Acknowledgements}

We wish to express our thanks to A. A. Iarrobino and P. M. Marques for inspiring conversations. Moreover we are also sincerely grateful to W. Buczyńska and J. Buczyński for their care, support and hospitality during the preparation of this paper. We also thank Buczyński for explaining the proof of Proposition 4.7. We thank the referee for careful reading and suggesting a number of improvements. The examples were obtained with the help of the Magma computing software; see [Bosma et al. 1997].

\section{References}

[Bernardi et al. 2011] A. Bernardi, A. Gimigliano, and M. Idà, "Computing symmetric rank for symmetric tensors”, J. Symbolic Comput. 46:1 (2011), 34-53. MR 2012h:14126 Zbl 1211.14057

[Bertone et al. 2012] C. Bertone, F. Cioffi, and M. Roggero, "A division algorithm in an affine framework for flat families covering Hilbert schemes”, preprint, 2012. arXiv 1211.7264

[Bosma et al. 1997] W. Bosma, J. Cannon, and C. Playoust, "The Magma algebra system, I: The user language”, J. Symbolic Comput. 24:3-4 (1997), 235-265. MR 1484478 Zbl 0898.68039

[Bruns and Herzog 1993] W. Bruns and J. Herzog, Cohen-Macaulay rings, Cambridge Studies in Advanced Mathematics 39, Cambridge University Press, 1993. MR 95h:13020 Zbl 0985.35075

[Buchsbaum and Eisenbud 1977] D. A. Buchsbaum and D. Eisenbud, "Algebra structures for finite free resolutions, and some structure theorems for ideals of codimension 3", Amer. J. Math. 99:3 (1977), 447-485. MR 56 \#11983 Zbl 0373.13006

[Buczyńska and Buczyński 2014] W. Buczyńska and J. Buczyński, "Secant varieties to high degree Veronese reembeddings, catalecticant matrices and smoothable Gorenstein schemes", J. Algebraic Geom. 23:1 (2014), 63-90. MR 3121848 Zbl 1295.14047

[Buczyński and Jelisiejew 2014] J. Buczyński and J. Jelisiejew, “On smoothability”, preprint, 2014, available at www.mimuw.edu.pl/ jjelisiejew/pdf/OnSmoothability06062014.pdf.

[Buczyński et al. $\geq 2015]$ J. Buczyński, T. Januszkiewicz, J. Jelisiejew, and M. Michałek, “On the existence of $k$-regular maps", submitted.

[Cartwright et al. 2009] D. A. Cartwright, D. Erman, M. Velasco, and B. Viray, "Hilbert schemes of 8 points", Algebra Number Theory 3:7 (2009), 763-795. MR 2011c:14010 Zbl 1187.14005

[Casnati and Notari 2009] G. Casnati and R. Notari, "On the Gorenstein locus of some punctual Hilbert schemes", J. Pure Appl. Algebra 213:11 (2009), 2055-2074. MR 2010g:14003 Zbl 1169.14003 
[Casnati and Notari 2011] G. Casnati and R. Notari, "On the irreducibility and the singularities of the Gorenstein locus of the punctual Hilbert scheme of degree 10", J. Pure Appl. Algebra 215:6 (2011), 1243-1254. MR 2012c:14007 Zbl 0837.58029

[Casnati and Notari 2014a] G. Casnati and R. Notari, "A structure theorem for 2-stretched Gorenstein algebras", preprint, 2014. To appear in J. Comm. Algebra. arXiv 1312.2191

[Casnati and Notari 2014b] G. Casnati and R. Notari, "On the Gorenstein locus of the punctual Hilbert scheme of degree 11", J. Pure Appl. Algebra 218:9 (2014), 1635-1651. MR 3188862 Zbl 1287.13013

[Casnati et al. 2014] G. Casnati, J. Jelisiejew, and R. Notari, "On the rationality of Poincarè series of Gorenstein algebras via Macaulay's correspondence”, preprint, 2014. To appear in Rocky Mountain J. Math. arXiv 1307.1676

[Eisenbud 1995] D. Eisenbud, Commutative algebra: with a view toward algebraic geometry, Graduate Texts in Mathematics 150, Springer, New York, 1995. MR 97a:13001 Zbl 0819.13001

[Elias and Rossi 2012] J. Elias and M. E. Rossi, "Isomorphism classes of short Gorenstein local rings via Macaulay's inverse system”, Trans. Amer. Math. Soc. 364:9 (2012), 4589-4604. MR 2922602 Zbl 1281.13015

[Elias and Rossi 2015] J. Elias and M. E. Rossi, "Analytic isomorphisms of compressed local algebras”, Proc. Amer. Math. Soc. 143:3 (2015), 973-987. MR 3293715 Zbl 1314.13041

[Elias and Valla 2011] J. Elias and G. Valla, "Isomorphism classes of certain Artinian Gorenstein algebras”, Algebr. Represent. Theory 14:3 (2011), 429-448. MR 2012c:13059 Zbl 1248.13022

[Ellingsrud 1975] G. Ellingsrud, "Sur le schéma de Hilbert des variétés de codimension 2 dans $\mathbb{P}^{e}$ à cône de Cohen-Macaulay”, Ann. Sci. École Norm. Sup. (4) 8:4 (1975), 423-431. MR 52 \#13831 Zbl 0325.14002

[Emsalem 1978] J. Emsalem, “Géométrie des points épais”, Bull. Soc. Math. France 106:4 (1978), 399-416. MR 80j:14008 Zbl 0396.13017

[Fogarty 1968] J. Fogarty, “Algebraic families on an algebraic surface”, Amer. J. Math 90 (1968), 511-521. MR 38 \#5778 Zbl 0176.18401

[Geramita 1999] A. V. Geramita, "Catalecticant varieties", pp. 143-156 in Commutative algebra and algebraic geometry (Ferrara), edited by F. van Oystaeyen, Lecture Notes in Pure and Appl. Math. 206, Dekker, New York, 1999. MR 2000f:14075 Zbl 0957.13007

[Grothendieck 1995] A. Grothendieck, "Techniques de construction et théorèmes d'existence en géométrie algébrique, IV: Les schémas de Hilbert", Exposé No. 221, 249-276 in Séminaire Bourbaki, vol. 6, Soc. Math. France, Paris, 1995. MR 1611822 Zbl 1031.00546

[Hartshorne 1966] R. Hartshorne, "Connectedness of the Hilbert scheme", Inst. Hautes Études Sci. Publ. Math. 29 (1966), 5-48. MR 35 \#4232 Zbl 0171.41502

[Hartshorne 1977] R. Hartshorne, Algebraic geometry, Graduate Texts in Mathematics 52, Springer, New York, 1977. MR 57 \#3116 Zbl 0367.14001

[Hartshorne 2010] R. Hartshorne, Deformation theory, Graduate Texts in Mathematics 257, Springer, New York, 2010. MR 2011c:14023 Zbl 1186.14004

[Iarrobino 1972] A. Iarrobino, "Reducibility of the families of 0-dimensional schemes on a variety", Invent. Math. 15 (1972), 72-77. MR 46 \#170 Zbl 0227.14006

[Iarrobino 1977] A. A. Iarrobino, Punctual Hilbert schemes, Mem. Amer. Math. Soc. 188, Amer. Math. Soc., Providence, RI, 1977. MR 58 \#5667 Zbl 0355.14001

[Iarrobino 1984] A. Iarrobino, "Compressed algebras: Artin algebras having given socle degrees and maximal length”, Trans. Amer. Math. Soc. 285:1 (1984), 337-378. MR 85j:13030 Zbl 0548.13009 
[Iarrobino 1994] A. A. Iarrobino, Associated graded algebra of a Gorenstein Artin algebra, Mem. Amer. Math. Soc. 514, Amer. Math. Soc., Providence, RI, 1994. MR 94f:13009 Zbl 0793.13010

[Iarrobino and Emsalem 1978] A. Iarrobino and J. Emsalem, "Some zero-dimensional generic singularities; finite algebras having small tangent space", Compositio Math. 36:2 (1978), 145-188. MR 81c:14004 Zbl 0393.14002

[Iarrobino and Kanev 1999] A. Iarrobino and V. Kanev, Power sums, Gorenstein algebras, and determinantal loci, Lecture Notes in Mathematics 1721, Springer, Berlin, 1999. MR 2001d:14056 Zbl 0942.14026

[Jelisiejew 2014a] J. Jelisiejew, "Deformations of zero-dimensional schemes and applications", preprint, 2014. arXiv 1307.8108

[Jelisiejew 2014b] J. Jelisiejew, "Local finite-dimensional Gorenstein $k$-algebras having Hilbert function $(1,5,5,1)$ are smoothable", J. Algebra Appl. 13:8 (2014), 1450056, 7. MR 3225123 Zbl 1317.13040

[Kleppe and Miró-Roig 1998] J. O. Kleppe and R. M. Miró-Roig, “The dimension of the Hilbert scheme of Gorenstein codimension 3 subschemes”, J. Pure Appl. Algebra 127:1 (1998), 73-82. MR 99a:14002 Zbl 0949.14003

[Kunz 2005] E. Kunz, Introduction to plane algebraic curves, Birkhäuser, Boston, 2005. MR 2006b: 14001 Zbl 1078.14041

[Landsberg and Ottaviani 2013] J. M. Landsberg and G. Ottaviani, "Equations for secant varieties of Veronese and other varieties", Ann. Mat. Pura Appl. (4) 192:4 (2013), 569-606. MR 3081636 Zbl 1274.14058

[Matsumura 1986] H. Matsumura, Commutative ring theory, Cambridge Studies in Advanced Mathematics 8, Cambridge University Press, 1986. MR 88h:13001 Zbl 0603.13001

[Miró-Roig 1992] R. M. Miró-Roig, "Nonobstructedness of Gorenstein subschemes of codimension 3 in $\mathbb{P}^{n}$ ", Beiträge Algebra Geom. 33 (1992), 131-138. MR 93d:14071 Zbl 0766.14001

[Raicu 2012] C. Raicu, "Secant varieties of Segre-Veronese varieties", Algebra Number Theory 6:8 (2012), 1817-1868. MR 3033528 Zbl 1273.14102

[Sally 1979] J. D. Sally, "Stretched Gorenstein rings", J. London Math. Soc. (2) 20:1 (1979), 19-26. MR 80k:14006 Zbl 0402.13018

[Stanley 1996] R. P. Stanley, Combinatorics and commutative algebra, 2nd ed., Progress in Mathematics 41, Birkhäuser, Boston, 1996. MR 98h:05001 Zbl 0838.13008

[Valabrega and Valla 1978] P. Valabrega and G. Valla, "Form rings and regular sequences", Nagoya Math. J. 72 (1978), 93-101. MR 80d:14010 Zbl 0362.13007

Communicated by Ravi Vakil

Received 2014-09-13 Revised 2015-04-18 Accepted 2015-06-17

gianfranco.casnati@polito.it Dipartimento di Scienze Matematiche, Politecnico di Torino, corso Duca degli Abruzzi 24, I-10129 Torino, Italy

j.jelisiejew@mimuw.edu.pl Faculty of Mathematics, Informatics, and Mechanics, University of Warsaw, Banacha 2, 02-097 Warsaw, Poland

roberto.notari@polimi.it Dipartimento di Matematica "Francesco Brioschi", Politecnico di Milano, via Bonardi 9, I-20133 Milano, Italy 


\section{Algebra \& Number Theory}

msp.org/ant

\section{EDITORS}

MANAGING EDITOR

Bjorn Poonen

Massachusetts Institute of Technology

Cambridge, USA

\author{
EDITORIAL BOARD CHAIR \\ David Eisenbud \\ University of California \\ Berkeley, USA
}

BOARD OF EDITORS

Georgia Benkart

Dave Benson

Richard E. Borcherds

John H. Coates

J-L. Colliot-Thélène

Brian D. Conrad

Hélène Esnault

Hubert Flenner

Sergey Fomin

Edward Frenkel

Andrew Granville

Joseph Gubeladze

Roger Heath-Brown

Craig Huneke

Kiran S. Kedlaya

János Kollár

Yuri Manin

Philippe Michel
University of Wisconsin, Madison, USA

University of Aberdeen, Scotland

University of California, Berkeley, USA

University of Cambridge, UK

CNRS, Université Paris-Sud, France

Stanford University, USA

Freie Universität Berlin, Germany

Ruhr-Universität, Germany

University of Michigan, USA

University of California, Berkeley, USA

Université de Montréal, Canada

San Francisco State University, USA

Oxford University, UK

University of Virginia, USA

Univ. of California, San Diego, USA

Princeton University, USA

Northwestern University, USA

École Polytechnique Fédérale de Lausanne
Susan Montgomery

Shigefumi Mori

Raman Parimala

Jonathan Pila

Anand Pillay

Victor Reiner

Peter Sarnak

Joseph H. Silverman

Michael Singer

Vasudevan Srinivas

J. Toby Stafford

Ravi Vakil

Michel van den Bergh

Marie-France Vignéras

Kei-Ichi Watanabe

Efim Zelmanov

Shou-Wu Zhang
University of Southern California, USA

RIMS, Kyoto University, Japan

Emory University, USA

University of Oxford, UK

University of Notre Dame, USA

University of Minnesota, USA

Princeton University, USA

Brown University, USA

North Carolina State University, USA

Tata Inst. of Fund. Research, India

University of Michigan, USA

Stanford University, USA

Hasselt University, Belgium

Université Paris VII, France

Nihon University, Japan

University of California, San Diego, USA

Princeton University, USA

\section{PRODUCTION}

production@msp.org

Silvio Levy, Scientific Editor

See inside back cover or msp.org/ant for submission instructions.

The subscription price for 2015 is US $\$ 255 /$ year for the electronic version, and $\$ 440 /$ year ( $+\$ 55$, if shipping outside the US) for print and electronic. Subscriptions, requests for back issues and changes of subscribers address should be sent to MSP.

Algebra \& Number Theory (ISSN 1944-7833 electronic, 1937-0652 printed) at Mathematical Sciences Publishers, 798 Evans Hall \#3840, c/o University of California, Berkeley, CA 94720-3840 is published continuously online. Periodical rate postage paid at Berkeley, CA 94704, and additional mailing offices.

ANT peer review and production are managed by EditFLOW ${ }^{\circledR}$ from MSP.

\section{PUBLISHED BY}

- mathematical sciences publishers

nonprofit scientific publishing

http://msp.org/

(C) 2015 Mathematical Sciences Publishers 


\section{Algebra \& Number Theory}

Volume $9 \quad$ No. $7 \quad 2015$

Singular moduli that are algebraic units

PHILIPP HABEGGER

Irreducibility of the Gorenstein loci of Hilbert schemes via ray families

GIANFRANCO CASNATI, JOACHIM JELISIEJEW and ROBERTO NOTARI

$p$-adic heights of Heegner points on Shimura curves

DANIEL DISEGNI

Calculabilité de la cohomologie étale modulo $\ell$

David A. Madore and FABrice ORgogozo 